\title{
FORCED OSCILLATIONS OF A DAMPED KORTEWEG-DE VRIES EQUATION IN A QUARTER PLANE
}

\author{
JERRY L. BONA \\ Department of Mathematics, Statistics $\&$ Computer Science, \\ University of Illinois at Chicago, Chicago, IL 60607, USA \\ bona@math.uic.edu \\ S. M. SUN \\ Department of Mathematics, \\ Virginia Polytechnic Institute and \\ State University, Blacksburg, Virginia 24061, USA \\ sun@math.vt.edu \\ BING-YU ZHANG \\ Department of Mathematical Sciences, \\ University of Cincinnati, Cincinnati, \\ Ohio 45221-0025, USA \\ bzhang@math.uc.edu
}

Received 19 November 2002

Laboratory experiments have shown that when nonlinear, dispersive waves are forced periodically from one end of an undisturbed stretch of the medium of propagation, the signal eventually becomes temporally periodic at each spatial point. It is our purpose here to establish this as a fact at least in the context of a damped Korteweg-de Vries equation. Thus, consideration is given to the initial-boundary-value problem

$$
\left.\begin{array}{l}
u_{t}+u_{x}+u u_{x}+u_{x x x}+\alpha u=0, \quad x \geq 0, t \geq 0, \\
u(0, t)=h(t), \quad u(x, 0)=\phi(x) .
\end{array}\right\}
$$

For this problem, it is shown that if the small amplitude, boundary forcing $h$ is periodic of period $T$, say, then the solution $u$ of $(*)$ is eventually periodic of period $T$. More precisely, we show for each $x>0$, that $u(x, t+T)-u(x, t)$ converges to zero exponentially as $t \rightarrow \infty$. Viewing $(*)$ (without the initial condition) as an infinite dimensional dynamical system in the Hilbert space $H^{s}\left(R^{+}\right)$for suitable values of $s$, we also demonstrate that for a given, small amplitude time-periodic boundary forcing, the system $(*)$ admits a unique limit cycle, or forced oscillation (a solution of $(*)$ without the initial condition that is exactly periodic of period $T$ ). Furthermore, we show that this limit cycle is globally exponentially stable. In other words, it comprises an exponentially stable attractor for the infinite-dimensional dynamical system described by $(*)$. 
Keywords: Wavemaker problem for a damped Korteweg-de Vries equation; forced oscilations; time periodic water waves; damped forced nonlinear evolution equations.

Mathematics Subject Classification 2000: 35Q53, 35B10, 35B40, 76B15

\section{Introduction}

In this paper we consider an initial-boundary problem for the Korteweg-de Vries equation posed in a quarter plane with a damping term appended, namely,

$$
\begin{array}{ll}
u_{t}+u_{x}+u u_{x}+u_{x x x}+\alpha u=0 & \text { for } x, t \geq 0, \\
u(x, 0)=\phi(x) & u(0, t)=h(t)
\end{array}
$$

where $\alpha>0$ is a constant that is proportional to the strength of the damping effect. Guided by the outcome of laboratory experiments, interest is given to the long term effect of the boundary forcing $h$.

In the experiments of Bona, Pritchard and Scott [1], a channel partly filled with water was mounted with a flap-type wavemaker at one end. Each experiment commenced with the water in the channel at rest. The wavemaker was activated and underwent periodic oscillations. The throw of the wavemaker and its frequency of oscillation was such that the surface waves brought into existence were of small amplitude and long wavelength, so candidates for description by Korteweg-de Vries type or BBM-type model equations. Two points about this set of experiments are especially relevant to the present discussion. First, it was clear from the experimental outcome that damping effects must be accounted in addition to those of nonlinearity and dispersion for good quantitative agreement with model predictions. When suitable dissipation was added, it appeared that these long-wave models provided an accurate description of reality for a reasonably wide range of amplitudes and frequencies. Second, it was observed at each fixed station down the channel, for example at a spatial point represented by $x_{0}$, that the wave motion $u\left(x_{0}, t\right)$, say, rapidly became periodic of the same period as the boundary forcing.

Our goal in the present paper is to consider the initial-boundary-value problem (1.1) with boundary forcing $h$ which is periodic of period $\theta$ and show that the resulting solution $u(x, t)$ is, for each fixed $x$, asymptotically periodic of period $\theta$. This program is carried out for the particular choice of damping effect appearing in (1.1a) and aims to establish as a fact that such a model predicts the interesting qualitative property observed in experiments and in associated numerical simulations. In a little more detail, it will be demonstrated that if the boundary forcing $h$ is small, a hypothesis consistent with the use of the Korteweg-de Vries description, and periodic of period $\theta$, then there is what we will call a forced oscillation solution $u^{*}(x, t)$ of (1.1a) that is temporally periodic of period $\theta$ and such that $u^{*}(0, t)=h(t)$ for $t>0$. Moreover, it is shown that the solution $u(x, t)$ of (1.1) converges to $u^{*}(x, t)$ in a sense to be made precise presently. If we conceive of 
(1.1) as a dynamical system, then this result is analogous to convergence to a limit cycle under periodic forcing. Uniqueness and stability of this limit cycle will also be discussed.

Damped KdV-equations have been studied in the past from the point of view of dynamical systems. Ghidaglia [9, 10] and Sell and You [14] considered the damped forced $\mathrm{KdV}$ equation

$$
\left.\begin{array}{l}
u_{t}+u u_{x}+u_{x x x}-\eta u_{x x}+\alpha u=f, \quad x \in(0,1), t \geq 0 \\
u(0, t)=u(1, t), \quad u_{x}(1, t)=u_{x}(0, t), \quad u_{x x}(1, t)=u_{x x}(0, t),
\end{array}\right\}
$$

posed on the finite interval $(0,1)$ with periodic boundary conditions, where $\alpha$ and $\eta$ are nonnegative constants and the forcing $f=f(x, t)$ is a function of $x$ and $t$. Assuming that $\eta=0, \alpha>0$ and that the external excitation $f$ is either timeindependent or time-periodic, Ghidaglia $[9,10]$ proved the existence of a global attractor of finite fractal dimension for the infinite dimensional system described by (1.2). Assuming $\eta>0$ Sell and You [14] showed that (1.2) possesses an inertial manifold in the case where the external excitation $f$ is time-independent.

In [18], Zhang studied a damped forced $\mathrm{KdV}$-equation posed on a finite interval with homogeneous Dirichlet boundary conditions, viz.

$$
\left.\begin{array}{l}
u_{t}+u u_{x}+u_{x x x}-\eta u_{x x}+u_{x}=f, \quad x \in(0,1), t \geq 0, \\
u(0, t)=0, \quad u(1, t)=0), \quad u_{x}(1, t)=0 .
\end{array}\right\}
$$

It was shown that if the external excitation $f$ is time periodic with small amplitude, then the system admits a unique time periodic solution which, as a limit cycle, forms an inertial manifold for the infinite dimensional system described by (1.3). Similar results have also been established by Zhang [19] for the damped BBM equation.

There have been many studies concerned with time-periodic solutions of partial differential equations in the literature. Early works on this subject include, for example, Brézis [5], Vejvoda et al. [15], Keller and Ting [7] and Rabinowitz [12, 13]. For recent theory, see Bourgain [4], Craig and Wayne [6] and Wayne [17]. In particular, in a nicely crafted and very accessible article [16] in the Notices of the AMS, Wayne has provided a very helpful review of theory pertaining to timeperiodic solutions of nonlinear partial differential equations. Most of the theory extant thus far has been in the context of parabolic or hyperbolic equations. Consideration of this issue for nonlinear dispersive equations is sparse, and the important question of stability of periodic solutions has received very little attention.

The paper is organized as follows. Section 2 is devoted to a brief review of notation and the function-analytic setting together with technical lemmas from the well-posedness theory for (1.1). The long-time behavior of solutions of (1.1) is investigated in Sec. 3. In particular, time-independent bounds on solutions are established, which are important for the main analysis that is developed in Sec. 4 . The outcome of our development is roughly the following. If $h$ is a periodic function 
on the half line $R^{+}$which is small enough in the Sobolev class $H^{s}\left(R^{+}\right)$, then there is a unique time-periodic solution $u^{*}(x, t)$ of (1.1a) associated to the boundary values $h(t)$, which, for each $t$, lies in $H^{s}\left(R^{+}\right)$. Moreover, this solution is shown in Sec. 5 to be either locally or globally exponentially stable depending on whether $s \in(3 / 4,1]$ or $s \geq 1$, respectively. The paper concludes with a short summary and suggestions for related lines of inquiry.

\section{Notation and Well-Posedness Results}

We begin with a review of terminology and notation. For an arbitrary Banach space $X$, the associated norm is denoted by $\|\cdot\|_{X}$. If $\Omega=(a, b)$ is a bounded open interval in $R^{+}=(0, \infty)$ and $k$ a non-negative integer, we denote by $C^{k}(\bar{\Omega})=C^{k}(a, b)$ the functions that, along with their first $k$ derivatives, are continuous on $[a, b]$ with the norm

$$
\|f\|_{C^{k}(\Omega)}=\sup _{x \in \Omega, 0 \leq j \leq k}\left|f^{(j)}(x)\right| .
$$

If $\Omega$ is an unbounded domain, $C_{b}^{k}(\bar{\Omega})$ is defined just as when $\Omega$ is bounded except that $f, f^{\prime}, \ldots, f^{(k)}$ are required to be bounded as well as continuous on $\bar{\Omega}$. The norm is defined as in (2.1). Similar definitions apply if $\Omega$ is an open set in $R^{N}$. The space $C^{\infty}(\bar{\Omega})=\cap_{j} C^{j}(\bar{\Omega})$ will appear tangentially, but its Frechet-space topology will not be needed. The space of test functions $\mathcal{D}(\Omega)$ is the usual subspace of $C^{\infty}(\bar{\Omega})$ consisting of functions with compact support in $\Omega$. Its dual space $\mathcal{D}^{\prime} \Omega$ ) is the space of Schwartz distributions on $\Omega$. For $1 \leq p \leq \infty, L_{p}(\Omega)$ connotes those functions $f$ which are $p$ th-power absolutely integrable on $\Omega$ with the usual modification in case $p=\infty$. If $k \geq 0$ is an integer and $1 \leq p \leq \infty$, let $W_{p}^{k}(\Omega)$ be the Sobolev space consisting of those $L_{p}(\Omega)$-functions whose first $k$ generalized derivatives lie in $L_{p}(\Omega)$, equipped with the usual norm

$$
\|f\|_{W_{p}^{k}(\Omega)}^{p}=\sum_{j=0}^{k}\left\|f^{(j)}\right\|_{L_{p}(\Omega)}^{p} .
$$

The case $p=2$ will appear frequently in our analysis; it is written in abbreviated form, viz. $H^{k}(\Omega)=W_{2}^{k}(\Omega)$. If $s \geq 0$ is a real number and $k \leq s<k+1$ for some integer $k$, then $H^{s}(\Omega)$ is the standard interpolation space of $H^{k}(\Omega)$ and $H^{k+1}(\Omega)$ as in [11]. In the analysis of the quarter-plane problem, the space $H^{s}(\Omega)$ will occur often with $\Omega=R^{+}$or $\Omega=(a, b)$. Because of their frequent occurrence, it is convenient to abbreviate their norms thusly;

$$
\|\cdot\|_{s}=\|\cdot\|_{H^{s}\left(R^{+}\right)} \quad \text { and } \quad|\cdot|_{s,(a, b)}=\|\cdot\|_{H^{s}(a, b)} .
$$

If $s=0$, the subscript $s$ will be omitted altogether, so that

$$
\|\cdot\|=\|\cdot\|_{L_{2}\left(R^{+}\right)} \quad \text { and } \quad|\cdot|_{(a, b)}=\|\cdot\|_{0,(a, b)} .
$$


(Norms on a finite interval, for example $(0, T)$ will usually be applied in the temporal variable, whereas norms on $R^{+}$will normally, but not universally, be applied in the spatial variable.) For $s \geq 0, H_{0}^{s}\left(R^{+}\right)$is the closure of $\mathcal{D}\left(R^{+}\right)$in the space $H^{s}\left(R^{+}\right)$ and $H_{\mathrm{loc}}^{s}(\Omega)$ is the set of measurable, real-valued functions $f$ defined on $\Omega$ such that for each $\phi \in \mathcal{D}(\Omega), \phi f \in H^{s}(\Omega)$. If $X$ is a Banach space, $T$ a positive real number and $1 \leq p \leq \infty$, denote by $L_{p}(0, T ; X)$ the Banach space of all measurable functions $u:(0, T) \rightarrow X$, such that $t \rightarrow\|u(t)\|_{X}$ is in $L_{p}(0, T)$, with norm

$$
\|u\|_{L_{p}(0, T ; X)}=\left(\int_{0}^{T}\|u(t)\|_{X}^{p} d t\right)^{1 / p}, \quad \text { if } 1 \leq p<\infty,
$$

and the obvious modification if $p=\infty$.

To discuss basic aspects of the well-posedness theory for the initial-boundaryvalue problem (IBVP henceforth) (1.1), some special semi-norms and Banach spaces will be used. We start by laying out helpful notation related to the fractional-order Sobolev classes defined on $R^{+}$. For $s \geq 0$, write $s=m+s^{\prime}$ where $0 \leq s^{\prime}<1$ and $m$ is a non-negative integer. Thus $m=[s]$, the greatest integer in $s$. For $f \in C^{\infty}\left(R^{+}\right) \cap H^{m}\left(R^{+}\right)$, define a new function $J_{x}^{s} f$ by

$$
J_{x}^{s} f(x)= \begin{cases}\left|f^{(m)}(x)\right| & \text { if } s^{\prime}=0, \\ \left(\int_{0}^{\infty} \tau^{-\left(2 s^{\prime}+1\right)}\left|f^{(m)}(x+\tau)-f^{(m)}(x)\right|^{2} d \tau\right)^{1 / 2} & \text { if } s^{\prime}>0,\end{cases}
$$

for any $x \in R^{+}$. Because $s^{\prime}<1$ and $f^{(m)}$ is smooth and an $L_{2}\left(R^{+}\right)$-function, $J_{x}^{s} f(x)$ is finite for all $x$. The quantity

$$
\|f\|_{H^{s}\left(R^{+}\right)}^{2}=\|f\|^{2}+\left\|J_{x}^{s} f\right\|^{2}
$$

defines a norm on $C^{\infty}\left(R^{+}\right) \cap H^{m}\left(R^{+}\right)$and the completion of this space in the norm (2.2) is another way of defining $H^{s}\left(R^{+}\right)$. (Remember, an unadorned norm is that of $L_{2}\left(R^{+}\right)$.) The space $H_{0}^{s}\left(R^{+}\right)$is the completion of $C_{0}^{\infty}\left(R^{+}\right)$in the norm defined in (2.2). Clearly $H_{0}^{s}\left(R^{+}\right)$is a closed linear subspace of $H^{s}\left(R^{+}\right)$and as is well known,

$$
H_{0}^{s}\left(R^{+}\right)=H^{s}\left(R^{+}\right)
$$

if $0 \leq s<1 / 2$.

For given $s \geq 0, b>a \geq 0$ and any function

$$
w \equiv w(x, t): R^{+} \times[a, b] \rightarrow R,
$$

define

$$
\begin{aligned}
& \Lambda_{1, s}^{(a, b)}(w) \equiv \sup _{a \leq t \leq b}\|w(\cdot, t)\|_{s} \\
& \Lambda_{2, s}^{(a, b)}(w) \equiv\left(\sup _{x \in R^{+}} \int_{a}^{b}\left|J_{x}^{s+1} w(x, t)\right|^{2} d t\right)^{1 / 2},
\end{aligned}
$$


if $s$ is an integer, and if $s$ is not an integer,

$$
\Lambda_{2, s}^{(a, b)}(w) \equiv\left(\sup _{x \in R^{+}} \int_{a}^{b} \int_{0}^{\infty} \tau^{-\left(2 s^{\prime}+1\right)}\left|D_{x}^{[s]+1} w(x+\tau, t)-D_{x}^{[s]+1} w(x, t)\right|^{2} d \tau d t\right)^{1 / 2}
$$

with $D_{x}=\frac{\partial}{\partial x}$ and $s^{\prime}=s-[s]$ as before;

$$
\begin{aligned}
& \Lambda_{3, s}^{(a, b)}(w) \equiv \sup _{x \in R^{+}}\|w(x, \cdot)\|_{H^{\frac{s+1}{3}}(a, b)}+\sup _{x \in R^{+}}\left\|D_{x} w(x, \cdot)\right\|_{H^{\frac{s}{3}}(a, b)} ; \\
& \Lambda_{4}^{(a, b)}(w) \equiv\left(\int_{a}^{b} \sup _{x \in R^{+}}\left|D_{x} w(x, t)\right|^{4} d t\right)^{1 / 4} ;
\end{aligned}
$$

and

$$
\Lambda_{5}^{(a, b)}(w)=\left(\int_{0}^{\infty} \sup _{t \in[a, b]}|w(x, t)|^{2} d x\right)^{1 / 2}
$$

In addition, let

$$
\lambda_{(a, b)}^{s}(w)=\max \left\{\Lambda_{1, s}^{(a, b)}(w), \Lambda_{2, s}^{(a, b)}(w), \Lambda_{3, s}^{(a, b)}(w)\right\}
$$

and

$$
\Gamma_{(a, b)}^{s}(w)=\lambda_{(a, b)}^{s}(w)+\Lambda_{4}^{(a, b)}(w)+\Lambda_{5}^{(a, b)}(w) .
$$

For any $b>a \geq 0$ and $s$ in the interval $0 \leq s \leq 7 / 2$, let $Y_{(a, b)}^{s}$ be the collection of all functions $u \in C\left([a, b] ; H^{s}\left(R^{+}\right)\right)$whose norm $\|u\|_{Y_{(a, b)}^{s}}$ is finite, where

$$
\|u\|_{Y_{(a, b)}^{s}}= \begin{cases}\lambda_{(a, b)}^{s}(u) & \text { if } 0 \leq s \leq 1 / 2 \\ \lambda_{(a, b)}^{s}(u)+\Lambda_{4}^{(a, b)}(u) & \text { if } 1 / 2<s \leq 3 / 4 \\ \Gamma_{(a, b)}^{s}(u) & \text { if } 3 / 4<s \leq 7 / 2 .\end{cases}
$$

For $s>7 / 2$, write $s$ in the form

$$
s=3 m+s^{\prime}
$$

with $m=[s / 3]$ or $m=[s / 3]-1$ and $1 / 2<s^{\prime} \leq 7 / 2$. For $a$ and $b$ as above and such a value of $s$, let $Y_{(a, b)}^{s}$ be the collection of all functions $u \in C^{m-1}\left([a, b] ; H^{3}\left(R^{+}\right)\right)$ with $\partial_{t}^{m} u \in C\left([a, b] ; H^{s^{\prime}}\left(R^{+}\right)\right)$satisfying

$$
\|u\|_{Y_{(a, b)}^{s}}=\left\|\partial_{t}^{m} u\right\|_{Y_{(a, b)}^{s^{\prime}}}+\sum_{k=0}^{m-1}\left\|\partial_{t}^{k} u\right\|_{Y_{(a, b)}^{3}}<+\infty .
$$

In addition, let $X_{(a, b)}^{s}$ be the product $H^{s}\left(R^{+}\right) \times H^{(s+1) / 3}(a, b)$. While the preceding may seem a little complicated, these norms are needed to catch the smoothing effects that accrue when (1.1) is solved. These weak smoothing effects are central to the well-posedness for smaller values of $s$.

Attention is now turned to well-posedness results for the IBVP (1.1). First of all, as in many initial-boundary-value problems, some compatibility conditions are 
needed between the initial data $\phi$ and the boundary value $h$. A simple computation shows that if $u$ is a $C^{\infty}$-smooth solution of (1.1) up to the boundary, then its initial data $u(x, 0)=\phi(x)$ and its boundary value $u(0, t)=h(t)$ must satisfy

$$
\phi_{k}(0)=h_{k}(0)
$$

for $k=0,1, \ldots$, where $h_{k}(t) \equiv h^{(k)}(t)$ is the $k$-th derivative of $h$,

$$
\left.\begin{array}{l}
\phi_{0}(x)=\phi(x), \quad \text { and } \\
\phi_{k}(x)=-\left(\phi_{k-1}^{\prime \prime \prime}(x)+\phi_{k-1}^{\prime}(x)+\alpha \phi_{k-1}(x)+\sum_{j=0}^{k-1}\left[\phi_{j}(x) \phi_{k-j-1}(x)\right]^{\prime}\right)
\end{array}\right\}
$$

for $k=1,2, \ldots$.

Definition 2.1 ( $s$-compatibility). Given $T>0$ and $s \geq 0$, we say that $(\phi, h) \in$ $X_{(0, T)}^{s}$ is $s$-compatible if

$$
\phi_{k}(0)=h_{k}(0)
$$

for $k=0,1, \ldots,[s / 3]-1$ when $s-3[s / 3] \leq 1 / 2$ and for $k=0,1, \ldots,[s / 3]$ when $s-3[s / 3]>1 / 2$.

When $\alpha=0$, which is the equation without damping, the IBVP (1.1) has been studied in [2] and is known to be well-posed in the space $H^{s}\left(R^{+}\right)$for $s>3 / 4$. In case $\alpha>0$, let

$$
v(x, t)=e^{-\alpha t} u(x, t)
$$

where $u$ is a solution of (1.1). It is straightforward to see that $v$ solves the IBVP

$$
\left.\begin{array}{l}
v_{t}+v_{x}+e^{\alpha t} v v_{x}+v_{x x x}=0 \\
v(x, 0)=\phi(x), \quad v(0, t)=e^{-\alpha t} h(t) .
\end{array}\right\}
$$

The same arguments used in [2] can be applied to (2.3) with very slight modification to obtain exactly the same well-posedness results. Consequently, the following wellposedness results hold for the IBVP (1.1) with $\alpha>0$.

Theorem 2.1 (Local well-posedness). Let $T>0$ and $s>3 / 4$ be given. Then for any s-compatible pair $(\phi, h) \in X_{(0, T)}^{s}$, there exists a $T^{*} \in(0, T]$ depending only on $\|(\phi, h)\|_{X_{(0, T)}^{s}}$ such that (1.1) with initial data $\phi$ and boundary data $h$ admits a unique solution

$$
u \in Y_{0, T^{*}}^{s} \cap C\left(\left[0, T^{*}\right] ; H^{s}\left(R^{+}\right)\right)
$$

with $\partial_{t}^{k} u \in C\left(\left[0, T^{*}\right] ; H^{s-3 k}\left(R^{+}\right)\right)$for $k=0,1, \ldots,[s / 3]$. Moreover, the corresponding solution map is analytic.

Theorem 2.2 (Global well-posedness). Let $T>0$ and $s \geq 1$ be given. Then for any s-compatible $(\phi, h) \in H^{s}\left(R^{+}\right) \times H^{(7+3 s) / 12}(0, T)$ when $1 \leq s \leq 3$ and for any $s$-compatible $(\phi, h) \in X_{(0, T)}^{s}$ when $s>3$, the problem (1.1) admits a unique solution $u \in Y_{(0, T)}^{s} \cap C\left([0, T] ; H^{s}\left(R^{+}\right)\right)$with $\partial_{t}^{k} u \in C\left([0, T] ; H^{s-3 k}\left(R^{+}\right)\right)$for $k=$ $0,1, \ldots,[s / 3]$. Moreover, the corresponding solution map is analytic. 


\section{Preliminaries}

In this section, a series of technical lemmas is developed which will find frequent use subsequently. The following two inequalities are basic and elementary.

Lemma 3.1. (i) For any nonnegative numbers a and $b$,

$$
a b \leq \epsilon a^{p}+C(\epsilon) b^{q}, \quad \text { if } \quad \frac{1}{p}+\frac{1}{q}=1,1<p, q<\infty
$$

where $C(\epsilon)=\frac{p-1}{p^{q} \epsilon^{1 /(p-1)}}$.

(ii) For any $f \in H^{1}\left(R^{+}\right), f \in C_{b}\left(R^{+}\right)$and

$$
\|f\|_{C_{b}\left(R^{+}\right)} \leq \sqrt{2}\|f\|^{1 / 2}\left\|f^{\prime}\right\|^{1 / 2} .
$$

Next, consideration is given to an abstract result about a sequence in a Banach space $X$ generated by iteration as follows:

$$
y_{n+1}=A y_{n}+F\left(y_{n}\right), \quad n=0,1,2, \ldots .
$$

Here, the linear operator $A$ is bounded from $X$ to $X$ with

$$
\left\|A y_{n}\right\|_{X} \leq \gamma\left\|y_{n}\right\|_{X}
$$

for some finite value $\gamma$ and all $n \geq 0$. The nonlinear function $F$ mapping $X$ to $X$ is such that there are constants $\beta_{1}$ and $\beta_{2}$ and a sequence $\left\{b_{n}\right\}_{n \geq 0}$ for which

$$
\left\|F\left(y_{n}\right)\right\|_{X} \leq \beta_{1}\left\|y_{n}\right\|_{X}+\beta_{2}\left\|y_{n}\right\|_{X}^{2}+b_{n}
$$

for all $n \geq 0$. The following two lemmas apply to such a sequence. These lemmas will find use in Secs. 4 and 5.

Lemma 3.2. If $\beta_{2}=0$ in (3.3) and $r=\gamma+\beta_{1}<1$, then the sequence $\left\{y_{n}\right\}_{n=0}^{\infty}$ defined by (3.1) satisfies

$$
\left\|y_{n+1}\right\|_{X} \leq r^{n+1}\left\|y_{0}\right\|_{X}+\frac{b^{*}}{1-r}
$$

for any $n \geq 1$, where $b^{*}=\sup _{n \geq 0} b_{n}$.

Proof. The assumptions imply

$$
\left\|y_{n+1}\right\|_{X} \leq \gamma\left\|y_{n}\right\|_{X}+\beta_{1}\left\|y_{n}\right\|_{X}+b_{n}
$$

for $n \geq 0$. In particular, it follows that

$$
\left\|y_{1}\right\|_{X} \leq \gamma\left\|y_{0}\right\|_{X}+\beta_{1}\left\|y_{0}\right\|_{X}+b_{0}
$$

so that

$$
\left\|y_{2}\right\|_{X} \leq \gamma\left\|y_{1}\right\|_{X}+\beta_{1}\left\|y_{1}\right\|_{X}+b_{1} \leq r^{2}\left\|y_{0}\right\|_{X}+r b_{0}+b_{1} .
$$

Proceeding inductively leads to the conclusion

$$
\left\|y_{n+1}\right\|_{X} \leq r^{n+1}\left\|y_{0}\right\|_{X}+\sum_{k=0}^{n} r^{k} b_{n-k}
$$

from which (3.4) follows since $r<1$. 
Lemma 3.3. If $\beta_{2} \neq 0$ in (3.3) and $r=\gamma+\beta_{1}<1$, then there exist $r_{1}$ with $0<r_{1}<1, \delta_{1}>0$ and $\delta_{2}>0$ such that if $\left\|y_{0}\right\|_{X}<\delta_{1}$ and $b_{n} \leq \delta_{2}$ for all $n \geq 0$, the sequence $\left\{y_{n}\right\}_{n=0}^{\infty}$ defined by (3.1) satisfies

$$
\left\|y_{n+1}\right\|_{X} \leq r_{1}^{n+1}\left\|y_{0}\right\|_{X}+\frac{b^{*}}{1-r}
$$

for any $n \geq 1$, where $b^{*}=\max _{n}\left\{b_{n}\right\}$.

Proof. Choose $\delta_{1}=\frac{1-r}{2 \beta_{2}}$ and $\delta_{2}=\frac{1-r}{2} \delta_{1}$. An inductive argument shows that if $\left\|y_{0}\right\|_{X} \leq \delta_{1}$, then

$$
\left\|y_{n}\right\|_{X} \leq \delta_{1}, \quad \text { for all } n \geq 1
$$

In consequence, we have

$$
\begin{aligned}
\left\|y_{n+1}\right\|_{X} & \leq r\left\|y_{n}\right\|_{X}+\beta_{2}\left\|y_{n}\right\|_{X}^{2}+b_{n} \\
& \leq \gamma\left\|y_{n}\right\|_{X}+\frac{1-r}{2}\left\|y_{n}\right\|_{X}+b_{n} \\
& \leq \frac{1+r}{2}\left\|y_{n}\right\|_{X}+b_{n} .
\end{aligned}
$$

From this inequality, (3.5) follows from Lemma 3.2 by choosing $r_{1}=\frac{1+r}{2}$. The proof is complete.

Back to more concrete issues, consider the linear IBVP associated with (1.1),

$$
\left.\begin{array}{l}
v_{t}+v_{x}+v_{x x x}+\alpha v=f, \quad \text { for } x, t \geq 0, \\
v(x, 0)=\phi(x), \quad v(0, t)=h(t) .
\end{array}\right\}
$$

Remark 3.4. If $v$ is a solution of (3.6), then $w(x, t)=v(x, t) e^{-\alpha t}$ solves the linear problem

$$
\left.\begin{array}{l}
w_{t}+w_{x}+w_{x x x}=e^{\alpha t} f, \quad \text { for } x, t \geq 0, \\
w(x, 0)=\phi(x), \quad w(0, t)=e^{\alpha t} h(t) .
\end{array}\right\}
$$

Consideration is directed to the homogeneous linear problem

$$
\left.\begin{array}{l}
u_{t}+u_{x}+u_{x x x}+\alpha u=0, \quad \text { for } x, t \geq 0, \\
u(x, 0)=\phi(x), \quad u(0, t)=0 .
\end{array}\right\}
$$

By semigroup theory, its solution may be obtained in the form

$$
u(t)=W_{c, \alpha}(t) \phi,
$$

where the spatial variable is suppressed and $W_{c, \alpha}(t)$ is the $C_{0}$-semigroup in the space $L_{2}\left(R^{+}\right)$generated by the operator

$$
A_{\alpha} f=-f^{\prime \prime \prime}-f^{\prime}-\alpha f
$$


with the domain

$$
\mathcal{D}\left(A_{\alpha}\right)=\left\{f \in H^{3}\left(R^{+}\right) \mid f(0)=0\right\} .
$$

Moreover, d'Alembert's formula allows one to formally write the solution of the inhomogeneous linear problem

$$
\left.\begin{array}{l}
u_{t}+u_{x}+u_{x x x}+\alpha u=f, \quad \text { for } x, t \geq 0 \\
u(x, 0)=0, \quad u(0, t)=0
\end{array}\right\}
$$

in the form

$$
u(t)=\int_{0}^{t} W_{c, \alpha}(t-\tau) f(\cdot, \tau) d \tau .
$$

According to [2] and Remark 3.4, $W_{c, \alpha}(t) \phi$ has the following explicit formula:

$$
W_{c, \alpha}(t) \phi(x)=\sum_{j=0}^{2} e^{-\alpha t}\left(U_{j}^{+}(t) \phi(x)+\overline{U_{j}^{+}(t) \phi(x)}\right)
$$

for any $\phi \in L_{2}\left(R^{+}\right)$, where

$$
\begin{aligned}
& U_{0}^{+}(t) \phi(x)=\frac{1}{2 \pi} \int_{1}^{\infty} e^{i \mu^{3} t-i \mu t} \int_{0}^{\infty} e^{i \mu(x-\xi)} \phi(\xi) d \xi d \mu \\
& U_{1}^{+}(t) \phi(x)=-\frac{1}{2 \pi} \int_{1}^{\infty} e^{i \mu^{3} t-i \mu t} e^{-\left(\frac{i \mu+\sqrt{3 \mu^{2}-4}}{2}\right) x} \int_{0}^{\infty} e^{-i \mu \xi} \phi(\xi) d \xi d \mu
\end{aligned}
$$

and

$$
U_{2}^{+}(t) \phi(x)=\frac{1}{2 \pi i} \int_{0}^{\infty} e^{-\mu^{3} t-\mu t} e^{-\left(\frac{\mu-i \sqrt{3 \mu^{2}+4}}{2}\right) x} \int_{0}^{\infty} e^{-\mu \xi} \phi(\xi) d \xi d \mu .
$$

Similarly we may write the solution of the non-homogeneous boundary value problem

$$
\left.\begin{array}{l}
u_{t}+u_{x}+u_{x x x}+\alpha u=0, \quad \text { for } x, t \geq 0, \\
u(x, 0)=0, \quad u(0, t)=h(t)
\end{array}\right\}
$$

as

$$
u(x, t)=\left[W_{b, \alpha}(t) h\right](x)=e^{-\alpha t}\left\{\left[U_{b}(t) h\right](x)+\overline{\left[U_{b}(t) h\right](x)}\right\}
$$

where, for $x, t \geq 0$,

$\left[U_{b}(t) h\right](x)=\frac{1}{2 \pi} \int_{1}^{\infty} e^{i \mu^{3} t-i \mu t} e^{-\left(\frac{\sqrt{3 \mu^{2}-4}+i \mu}{2}\right) x}\left(3 \mu^{2}-1\right) \int_{0}^{\infty} e^{-\left(\mu^{3} i-i \mu\right) \xi} e^{\alpha \xi} h(\xi) d \xi d \mu$.

For $W_{c, \alpha}(t) \phi$ and $W_{b, \alpha}(t) h$, the following estimates hold (see [2]).

Lemma 3.5. For any $s \in[0,7 / 2]$ and any $\phi \in H_{0}^{s}\left(R^{+}\right)$if $0 \leq s \leq 1$ or $\phi \in$ $H_{0}^{1}\left(R^{+}\right) \cap H^{s}\left(R^{+}\right)$if $s>1$, there exists a constant $C$ independent of $\phi$ such that

$$
\left\|W_{c, \alpha}(t) \phi\right\|_{s} \leq C e^{-\alpha t}\|\phi\|_{s}
$$

for any $t \geq 0$. 
Lemma 3.6. Given $s \in[0,7 / 2]$ and $T>0$, there exists a constant $C=C_{s, T}$ such that

$$
\sup _{0 \leq t \leq T}\left\|\left[W_{b, \alpha}(t) h\right](\cdot)\right\|_{s} \leq C|h|_{\frac{s+1}{3},(0, T)}
$$

for all $h \in H_{\mathrm{loc}}^{\frac{s+1}{3}}\left(R^{+}\right)$with $h(0)=0$.

In terms of $W_{c, \alpha}(t)$ and $W_{b, \alpha}(t)$, the solution $v(x, t)$ of (3.6) may be written in the form

$$
\begin{aligned}
v(x, t)= & W_{c, \alpha}(t)\left(\phi(x)-e^{-x} \phi(0)\right)+\int_{0}^{t} W_{c, \alpha}(t-\tau)\left(f(x, \tau)+2 e^{-x-\tau} h(0)\right) d \tau \\
& +\left[W_{b, \alpha}(t)\left(h(t)-e^{-t} h(0)\right)\right](x)+e^{-x-t} h(0)
\end{aligned}
$$

provided $\phi \in H^{s}\left(R^{+}\right)$and $h \in H_{\mathrm{loc}}^{(s+1) / 3}\left(R^{+}\right)$with $s>1 / 2$. When $0 \leq s \leq 1 / 2$, the solution $v(x, t)$ of $(3.6)$ is simply

$$
v(x, t)=W_{c, \alpha}(t) \phi(x)+\int_{0}^{t} W_{c, \alpha}(t-\tau) f(x, \tau) d \tau+\left[W_{b, \alpha}(t) h(t)\right](x) .
$$

The next four results are taken directly from [2]. They are central to the later developments and are repeated here for the reader's convenience.

Lemma 3.7. Let $s \geq 0, T>0$ and $\epsilon>0$ be given. There exists a constant $C$ depending only on $s, T$ and on $\epsilon$ when it appears, such that

(i) for $0 \leq s \leq 1 / 2$,

$$
\lambda_{(0, T)}^{s}(v) \leq C\left(\|\phi\|_{s}+|h|_{\frac{s+1}{3},(0, T)}+\int_{0}^{T}\|f(\cdot, t)\|_{s} d t\right)
$$

(ii) for $1 / 2<s \leq 2$ and $\phi(0)=h(0)$,

$$
\lambda_{(0, T)}^{s}(v) \leq C\left(\|\phi\|_{s}+|h|_{\frac{s+1}{3},(0, T)}+|f(0, \cdot)|_{(0, T)}+\int_{0}^{T}\|f(\cdot, t)\|_{s} d t\right) ;
$$

(iii) for $2 \leq s \leq 3$ and $\phi(0)=h(0)$,

$$
\lambda_{(0, T)}^{s}(v) \leq C\left(\|\phi\|_{s}+|h|_{\frac{s+1}{3},(0, T)}+|f(0, \cdot)|_{\frac{s-2}{3},(0, T)}+\int_{0}^{T}\|f(\cdot, t)\|_{s} d t\right) ;
$$

(iv) if $\phi(0)=h(0)$, then

$$
\begin{aligned}
\Lambda_{4}^{(0, T)}(v) \leq & C\left(\|\phi\|_{\frac{1}{2}}+\|h\|_{\frac{1}{2},(0, T)}+\int_{0}^{T}\|f(\cdot, t)\|_{\frac{1}{2}} d t\right) \\
& +C\left(|\phi(0)|+|f(0, \cdot)|_{(0, T)}\right)
\end{aligned}
$$


(v) if $\phi(0)=h(0)$, then

$$
\begin{aligned}
\Lambda_{5}^{(0, T)}(v) \leq & C\left(\|\phi\|_{\frac{3}{4}+\epsilon}+|h|_{\frac{7}{12}+\epsilon,(0, T)}+\int_{0}^{T}\|f(\cdot, t)\|_{\frac{3}{4}+\epsilon} d t\right) \\
& +C|f(0, \cdot)|_{(0, T)} .
\end{aligned}
$$

Lemma 3.8. Let $0 \leq s \leq 7 / 2$ be given. Let $f(x, t)=e^{-x} h(t)$ where $h \in L_{2}\left(R^{+}\right)$ if $0 \leq s \leq 2$ and $h \in H^{(s-2) / 3}\left(R^{+}\right)$if $2<s \leq 7 / 2$. Then there exists a constant $C$ such that the function $u$ given by

$$
u(x, t)=\int_{0}^{t} W_{c, \alpha}(t-\tau) f(\cdot, \tau) d \tau
$$

obeys the inequalities

$$
\begin{aligned}
\sup _{0 \leq t<\infty} & \|u(\cdot, t)\|_{s}+\left(\sup _{x \in R^{+}}\left\|J_{x}^{s+1} u(x, \cdot)\right\|^{2}\right)^{1 / 2}+\sum_{k=0}^{1} \sup _{x \in R^{+}}\left\|D_{x}^{k} u(x, \cdot)\right\|_{H} \frac{s-k+1}{3}\left(R^{+}\right) \\
& +\left(\int_{0}^{\infty}\left\|\mathcal{D}^{s+1 / 4} u(\cdot, t)\right\|_{L_{\infty}\left(R^{+}\right)}^{4} d t\right)^{1 / 4}+\left(\int_{0}^{\infty} \sup _{t \in[0, T]}|u(x, t)|^{2} d x\right)^{1 / 2} \\
\leq & \begin{cases}C\|h\| & \text { for } 0 \leq s \leq 2, \\
C\|h\|_{\frac{s-2}{3}} & \text { for } 2<s \leq 7 / 2 .\end{cases}
\end{aligned}
$$

For the space $Y_{(0, T)}^{s}$ defined in Sec. 2, the following bilinear estimates were established in [2]. These will also find use later.

Lemma 3.9. Let $T>0$ be given.

(i) If $3 / 4<s \leq 7 / 2$ and $u, v \in Y_{(0, T)}^{s}$, then $u v_{x} \in L_{2}\left(0, T ; H^{s}\left(R^{+}\right)\right)$and

$$
\left\|u v_{x}\right\|_{L_{2}\left(0, T ; H^{s}\left(R^{+}\right)\right)} \leq C\|u\|_{Y_{(0, T)}^{s}}\|v\|_{Y_{(0, T)}^{s}}
$$

where $C$ depends on $s$, but is independent of $T, u$ and $v$.

(ii) If $s \in[0,3 / 4], u \in Y_{(0, T)}^{1}$ and $v \in Y_{(0, T)}^{s}$, then $(u v)_{x} \in L_{2}\left(0, T ; H^{s}\left(R^{+}\right)\right)$and

$$
\left\|(u v)_{x}\right\|_{L_{2}\left(0, T ; H^{s}\left(R^{+}\right)\right)} \leq C\|u\|_{Y_{(0, T)}^{1}}\|v\|_{Y_{(0, T)}^{s}},
$$

where $C$ depends on $s$, but is independent of $T, u$ and $v$.

Finally, we consider the initial-boundary-value problem for a linearized, damped $\mathrm{KdV}$-equation, namely

$$
\left.\begin{array}{l}
u_{t}+u_{x}+(v u)_{x}+u_{x x x}+\alpha u=g, \quad x>0, \quad t>0, \\
u(x, 0)=\phi(x), \quad u(0, t)=h(t)
\end{array}\right\}
$$

where $v(x, t)$ is a given function. For this linearized system, the following conclusion is established in [2, Proposition 6.1]. 
Proposition 3.10. Let $T>0$ and $s \in(3 / 4,7 / 2]$ be given. Assume that $v \in Y_{(0, T)}^{s}$. Then, for any s-compatible pair $(\phi, h) \in X_{(0, T)}^{s}$ and $g \in L_{1}\left(0, T ; H^{s}\left(R^{+}\right)\right)$, the IBVP (3.8) admits a unique solution $u \in Y_{(0, T)}^{s}$ satisfying

$$
\|u\|_{Y_{(0, T)}^{s}} \leq \mu\left(\|v\|_{Y_{(0, T)}^{s}}\right)\left(\|(\phi, h)\|_{X_{(0, T)}^{s}}+\|g\|_{L_{1}\left(0, T ; H^{s}\left(R^{+}\right)\right)}\right)
$$

where $\mu: R^{+} \rightarrow R^{+}$is a T-dependent and continuous non-decreasing function which is independent of $(\phi, h)$ and $g$.

Proposition 3.11. Let $s \in(3 / 4,7 / 2]$ be given. There exist $T>0, r>0$ and $\beta>0$ such that if $\phi \in H^{s}\left(R^{+}\right)$and $h \in H_{\mathrm{loc}}^{\frac{s+1}{3}}\left(R^{+}\right)$are $s$-compatible and if

$$
g \in L_{1, \mathrm{loc}}\left(0, \infty ; H^{s}\left(R^{+}\right)\right), \quad v \in C\left(R^{+} ; H^{s}(R+)\right)
$$

satisfy

$$
\sup _{n \geq 0}\left(\|v\|_{Y_{(n T, n+1) T)}^{s}}+\|g\|_{L_{1}\left(n T,(n+1) T ; H^{s}\left(R^{+}\right)\right)}\right) \leq \beta
$$

then the solution $u$ of (3.8) satisfies

$$
\|u(\cdot, t)\|_{s} \leq C_{1} e^{-r t}\|\phi\|_{s}+C_{2} \sup _{n \geq 0}\left(|h|_{\frac{s+1}{3},(n T,(n+1) T)}+\|g\|_{L_{1}\left(n T,(n+1) T ; H^{s}\left(R^{+}\right)\right)}\right)
$$

for any $t \geq 0$, where $C_{1}$ and $C_{2}$ are constants independent of $(\phi, h), g$ and $t \in[0, T]$.

Remark 3.12. If $v \in C\left(R^{+} ; H^{1}\left(R^{+}\right)\right)$and (3.9) is replaced by

$$
\sup _{n \geq 0}\left(\|v\|_{Y_{(n T, n+1) T)}^{1}}+\|g\|_{L_{1}\left(n T,(n+1) T ; H^{s}\left(R^{+}\right)\right)}\right) \leq \beta
$$

then Proposition 3.11 holds for $0 \leq s \leq 3 / 4$. Note particularly the assumptions on $v$ and $g$ are local in time whereas the conclusion about $u$ is global in time. This is the crux of the matter.

Proof of Proposition 3.11. Using formula (3.7), the solution $u$ of (3.8) may be written as

$$
\begin{aligned}
u(x, t)= & W_{c, \alpha}(t) \phi_{1}(x)+\left[W_{b, \alpha}(t) h_{1}\right](x)+e^{-x-t} h(0) \\
& +\int_{0}^{t} W_{c, \alpha}(t-\tau)\left(f_{1}(x, \tau)+e^{-x} f(0, \tau)+2 e^{-x-\tau} h(0)+g(x, \tau)\right) d \tau
\end{aligned}
$$

with

$$
\phi_{1}(x)=\phi(x)-e^{-x} \phi(0), \quad f(x, t)=-(v(x, t) u(x, t))_{x}
$$

and

$$
f_{1}(x, t)=f(x, t)-e^{-x} f(0, t), \quad h_{1}(t)=h(t)-e^{-t} h(0) .
$$

By Lemma 3.5,

$$
u_{1}(x, t) \equiv W_{c, \alpha}(t) \phi_{1}(x)
$$


satisfies

$$
\left\|u_{1}(\cdot, t)\right\|_{s} \leq C e^{-\alpha t}\|\phi\|_{s}
$$

for any $t \geq 0$, where $C$ is a constant only depending on $s$. Using Lemmas 3.7 and 3.8 , it is adduced that there is a constant $C$ such that if

$$
u_{2}(x, t) \equiv \int_{0}^{t} W_{c, \alpha}(t-\tau)\left(f_{1}(x, \tau)+e^{-x} f(0, \tau)+2 e^{-x-\tau} h(0)+g(x, \tau)\right) d \tau,
$$

then

$$
\begin{aligned}
\left\|u_{2}(\cdot, t)\right\|_{s} \leq & C \int_{0}^{T}\left\|(v(\cdot, \tau) u(\cdot, \tau))_{x}\right\|_{s} d \tau+\int_{0}^{T}\|(g(\cdot, \tau))\|_{s} d \tau \\
& +C|h|_{\frac{s+1}{3},(0, T)}+C|q|_{\lambda(s),(0, T)}
\end{aligned}
$$

for any $t \in(0, T)$, where $q(t)=u_{x}(0, t) v(0, t)+v_{x}(0, t) u(0, t)$ and

$$
\lambda(s)= \begin{cases}0 & \text { if } s \leq 2 \\ (s-2) / 3 & \text { if } s \geq 2 .\end{cases}
$$

For the quantity

$$
u_{3}(x, t) \equiv\left[W_{b, \alpha}(t) h_{1}\right](x),
$$

Lemma 3.6 yields a constant $C$ for which

$$
\left\|u_{3}(\cdot, t)\right\|_{s} \leq C|h|_{\frac{s+1}{3},(0, T)}
$$

for any $t \in(0, T)$. As for

$$
u_{4}(x, t) \equiv e^{-x-t} h(0),
$$

it is clear that

$$
\left\|u_{4}(\cdot, t)\right\|_{s} \leq C|h|_{\frac{s+1}{3},(0, T)}
$$

for any $t \in(0, T)$. Moreover, it follows from Lemma 3.9 that

$$
\begin{aligned}
\int_{0}^{T}\left\|(v(\cdot, \tau) u(\cdot, \tau))_{x}\right\|_{s} d \tau & \leq T^{1 / 2}\left\|(v u)_{x}\right\|_{L_{2}\left(0, T ; H^{s}\left(R^{+}\right)\right)} \\
& \leq C T^{1 / 2}\|u\|_{Y_{(0, T)}^{s}}\|v\|_{Y_{(0, T)}^{s}} .
\end{aligned}
$$

In addition, for $3 / 4<s \leq 2$, it is true that

$$
\begin{aligned}
\left(\int_{0}^{T}\left|u_{x}(0, t) v(0, t)\right|^{2} d t\right)^{1 / 2} & =\left(\int_{0}^{T}\left|u_{x}(0, t)\right|^{2} d t \sup _{0 \leq t \leq T}|v(0, t)|^{2}\right)^{1 / 2} \\
& \leq C\left(\int_{0}^{T}\left|u_{x}(0, t)\right|^{2} d t\right)^{1 / 2} \sup _{0 \leq t \leq T}\|v(\cdot, t)\|_{s} \\
& \leq C T^{1 / 4}\|u\|_{Y_{(0, T)}^{s}}\|v\|_{Y_{(0, T)}^{s}} .
\end{aligned}
$$


On the other hand, if $2<s \leq 7 / 2$, then

$$
\begin{aligned}
\left|u_{x}(0, \cdot) v(0, \cdot)\right|_{\frac{s-2}{3},(0, T)} & \leq C T^{\frac{5-s}{6}}\left|u_{x}(0, \cdot) v(0, \cdot)\right|_{\frac{s+4}{9},(0, T)} \\
& \leq C T^{\frac{5-s}{6}}\|u\|_{Y_{(0, T)}^{s}}\|v\|_{Y_{(0, T)}^{s}} .
\end{aligned}
$$

Furthermore, by Proposition 3.10,

$$
\|u\|_{Y_{(0, T)}^{s}} \leq \mu\left(\|v\|_{Y_{(0, T)}^{s}}\right)\left(\|\phi\|_{s}+|h|_{\frac{s+1}{3},(0, T)}+\|g\|_{L_{1}\left(0, T ; H^{s}\left(R^{+}\right)\right)}\right),
$$

where $\mu: R^{+} \rightarrow R^{+}$. Combining the above estimates yields that for given $s \in$ $(3 / 4,7 / 2]$ and $T>0$, there exist constants $C_{j}(j=1,2,3)$ depending only on $T$ and $s$ such that

$$
\begin{aligned}
\|u(\cdot, T)\|_{s} \leq & C_{1} e^{-\alpha T}\|\phi\|_{s}+C_{2}\|v\|_{Y_{(0, T)}^{s}} \mu\left(\|v\|_{Y_{(0, T)}^{s}}\right)\|\phi\|_{s} \\
& +C_{3}\left(1+\mu\left(\|v\|_{Y_{(0, T)}^{s}}\right)\right)\left(|h|_{\frac{s+1}{3},(0, T)}+\|g\|_{L_{1}\left(0, T ; H^{s}\left(R^{+}\right)\right)}\right) .
\end{aligned}
$$

Let $y_{n}=u(\cdot, n T)$ for $n=0,1,2, \ldots$ and let $w$ be the solution of the IBVP

$$
\left.\begin{array}{l}
w_{t}+w_{x}+\left(v_{1} w\right)_{x}+w_{x x x}+\alpha w=g, \quad x \in R^{+}, 0 \leq t \leq T, \\
w(x, 0)=y_{n}(x), \quad w(0, t)=h(t+n T)
\end{array}\right\}
$$

with $v_{1}(x, t)=v(x, t+n T)$. Then $y_{n+1}(x)=w(x, T)$ by the semigroup property of the system (3.8). Consequently, we have the following estimate for $y_{n+1}$ :

$$
\begin{aligned}
\left\|y_{n+1}\right\|_{s} \leq & C_{1} e^{-\alpha T}\left\|y_{n}\right\|_{s}+C_{2}\|v\|_{Y_{(n T,(n+1) T)}^{s}} \mu\left(\|v\|_{Y_{(n T,(n+1) T)}^{s}}\right)\left\|y_{n}\right\|_{s} \\
& +C_{3}\left(1+\mu\left(\|v\|_{\left.Y_{(n T,(n+1) T)}^{s}\right)}\right)\left(|h|_{\frac{s+1}{3},(n T,(n+1) T)}+\|g\|_{L_{1}\left(n T,(n+1) T ; H^{s}\left(R^{+}\right)\right)}\right)\right.
\end{aligned}
$$

for any $n \geq 0$. Choose $T$ and $\beta$ such that

$$
C_{1} e^{-\alpha T}=\gamma<1
$$

and

$$
\gamma+C_{2} \beta_{1} \mu(\beta) \equiv r<1
$$

Then

$$
\left\|y_{n+1}\right\|_{s} \leq r\left\|y_{n}\right\|_{s}+b_{n}
$$

for $n \geq 0$ provided that

$$
\sup _{n \geq 0}\|v\|_{Y_{(n T,(n+1) T)}^{s}} \leq \beta
$$

where

$$
b_{n}=C_{3}\left(1+\mu\left(\|v\|_{Y_{(n T,(n+1) T)}^{s}}\right)\right)\left(|h|_{\frac{s+1}{3},(n T,(n+1) T)}+\|g\|_{L_{1}\left(n T,(n+1) T ; H^{s}\left(R^{+}\right)\right)}\right) .
$$

It follows from Lemma 3.2 that

$$
\left\|y_{n+1}\right\|_{s} \leq r^{n+1}\left\|y_{0}\right\|_{s}+\frac{b^{*}}{1-r}
$$

for any $n \geq 1$ where $b^{*}=\max _{n}\left\{b_{n}\right\}$. This inequality implies the conclusion of Proposition 3.11 . 


\section{Asymptotic Bounds}

In this section, attention is turned to the long-time behavior of solutions of (1.1). As a first step, interest is directed to whether or not the solutions are bounded uniformly in $t$ in the space $H^{s}\left(R^{+}\right)$.

The well-posedness result in Theorem 2.1 for the IBVP (1.1) is temporally local in the sense that given $s$-compatible auxiliary data $\phi$ and $h$, the corresponding solution $u$ is only guaranteed to exist on the time interval $\left(0, T^{*}\right)$, where $T^{*}$ depends on the norm of $(\phi, h)$ in the space $X_{(0, T)}^{s}$. Here, an alternative view of local wellposedness for the IBVP (1.1) is presented. The idea is that for given $s$ and $T>0$, if the norm of $(\phi, h)$ in the space $X_{(0, T)}^{s}$ is not too large, then the corresponding solution is guaranteed to exist over the entire time interval $(0, T)$.

Proposition 4.1. Let $T>0$ and $s>3 / 4$ be given. There exists a $\delta=\delta_{T, s}>0$ such that for any pair of $s$-compatible functions $(\phi, h) \in X_{(0, T)}^{s}$ satisfying

$$
\|(\phi, h)\|_{X_{(0, T)}^{s}} \leq \delta
$$

the IBVP (1.1) admits a unique solution $u \in Y_{(0, T)}^{s}$. Moreover,

$$
\|u\|_{Y_{(0, T)}^{s}} \leq C\|(\phi, h)\|_{X_{(0, T)}^{s}}
$$

where $C$ is a constant independent of $T, \phi$ and $h$.

Proof. The proof is a modification of the proof of [2, Theorem 4.8]. We content ourselves with a sketch and refer readers to [2] for more detail.

Let $\delta$ and $r$ be two positive constants to be determined later. For any element $(\phi, h) \in X_{(0, T)}^{s}$ which is an $s$-compatible pair with

$$
\|(\phi, h)\|_{X_{(0, T)}^{s}} \leq \delta,
$$

define the closed subset $S_{\delta, r}$ of $Y_{(0, T)}^{s}$ to be

$$
S_{\delta, r}=\left\{w \in Y_{(0, T)}^{s}: w(0, t)=h(t), \quad w(x, 0)=\phi(x), \quad\|w\|_{Y_{(0, T)}^{s}} \leq r\right\} .
$$

According to Proposition 3.11, for any $v \in S_{\delta, r}$, the linear problem

$$
\left.\begin{array}{l}
u_{t}+u_{x}+u_{x x x}+\alpha u=-v v_{x}, \quad \text { for } x, t \geq 0, \\
u(x, 0)=\phi(x), \quad u(0, t)=h(t)
\end{array}\right\}
$$

has a unique solution $u \in Y_{(0, T)}^{s}$. Thus there is defined a map $\Lambda$ from $S_{\delta, r}$ to $Y_{(0, T)}^{s}$, say

$$
u=\Lambda(v)
$$

for $v \in S_{\delta, r}$. Moreover, on account of our previous estimates,

$$
\|\Lambda(v)\|_{Y_{(0, T)}^{s}} \leq C\left(\|\phi\|_{s}+|h|_{\frac{s+1}{3},(0, T)}+\left(T^{1 / 4}+T^{1 / 2}\right)\|v\|_{Y_{(0, T)}^{s}}^{2}\right)
$$


for $s>3 / 4$. Here $C$ is independent of $\phi, h$ and $T$. Setting

$$
r=2 C\|(\phi, h)\|_{X_{(0, T)}^{s}}
$$

and choosing $\delta>0$ such that

$$
2 C^{2} \delta\left(T^{1 / 4}+T^{1 / 2}\right) \leq 1 / 2,
$$

it is seen immediately that

$$
\|\Lambda(v)\|_{Y_{(0, T)}^{s}} \leq r \quad \text { for any } v \in S_{\delta, r} .
$$

Thus $\Lambda$ is a map from $S_{\delta, r}$ to $S_{\delta, r}$ if $\delta$ and $r$ are chosen according to (4.1) and (4.2). A similar argument shows that for such $\beta$ and $r$,

$$
\left\|\Lambda\left(v_{1}\right)-\Lambda\left(v_{2}\right)\right\|_{Y_{(0, T)}^{s}} \leq \frac{1}{2}\left\|v_{1}-v_{2}\right\|_{Y_{(0, T)}^{s}}
$$

for any $v_{1}, v_{2} \in S_{\delta, r}$. Thus $\Lambda$ is a contraction from $S_{\delta, r}$ to $S_{\delta, r}$. Its unique fixed point $u$ is the desired solution of (1.1); it is defined on the temporal interval $[0, T]$ and satisfies

$$
\|u\|_{Y_{(0, T)}^{s}} \leq r=2 C\|(\phi, h)\|_{X_{(0, T)}^{s}} .
$$

The proof is complete.

Next, it is shown that if $\delta$ is small enough, then the corresponding solution $u$ of (1.1) exists for any $t>0$ and its norm in the space $H^{s}\left(R^{+}\right)$is uniformly bounded.

Theorem 4.2. Let $s>3 / 4$ be given. There exist positive constants $T, \beta_{j}(j=1,2)$, $r$ with $0<r<1$ and $C_{j}(j=1,2)$ such that for any $s$-compatible pair $(\phi, h) \in$ $H^{s}\left(R^{+}\right) \times H_{\mathrm{loc}}^{(s+1) / 3}\left(R^{+}\right)$with

$$
\|\phi\|_{s} \leq \beta_{1} \quad \text { and } \quad \sup _{n \geq 0}|h|_{\frac{s+1}{3},(n T,(n+1) T)} \leq \beta_{2},
$$

then the solution $u$ of the IBVP (1.1) is globally defined and belongs to the space $C_{b}\left(0, \infty ; H^{s}\left(R^{+}\right)\right)$. Moreover,

$$
\|u(\cdot, t)\|_{s} \leq C_{1} e^{-r t}\|\phi\|_{s}+C_{2} \sup _{n \geq 0}|h|_{\frac{s+1}{3},(n T,(n+1) T)}
$$

for all $t \geq 0$.

Remark 4.3. The important point here is that the assumed bound on the boundary forcing $h$ is only local in $t$ whereas the conclusion is global. As in Proposition 3.11, the damping term is crucial to the conclusion as formulated here for spaces that are not local in space.

Proof. First, consider the case $3 / 4<s \leq 7 / 2$ and fix an $s$ in this range. Assume temporarily that $(\phi, h)$ are $s$-compatible and that $\phi \in H^{7 / 2}\left(R^{+}\right)$and $h \in H_{\text {loc }}^{3 / 2}\left(R^{+}\right)$ so that by Theorem 2.2 the associated solution $u$ is globally defined in $R^{+} \times R^{+}$. Because of this, we need not be concerned about time intervals of existence. The 
resulting bounds do not depend on the additional regularity and, therefore, the continuous dependence result implies they are valid, so long as the solution exists, only assuming $(\phi, h) \in X_{(0, T)}^{s}$. Moreover, the bounds thereby derived, which are those displayed in the Theorem, suffice to iterate the local existence result with a constant time step, thereby obtaining globally defined solutions provided only that $s>3 / 4$.

For $(\phi, h) \in H^{s}\left(R^{+}\right) \times H_{\mathrm{loc}}^{(s+1) / 3}\left(R^{+}\right)$with $\phi(0)=h(0)$, the corresponding solution $u$ of (1.1) can be written in the form

$$
\begin{aligned}
u(x, t)= & W_{c, \alpha}(t) \phi_{1}(x)+\int_{0}^{t} W_{c, \alpha}(t-\tau)\left(f_{1}(x, \tau)+e^{-x} f(0, \tau)+2 e^{-x-\tau} h(0)\right) d \tau \\
& +\left[W_{b, \alpha}(t) h_{1}\right](x)+e^{-x-t} h(0)
\end{aligned}
$$

with

$$
\phi_{1}(x)=\phi(x)-e^{-x} \phi(0), \quad f(x, t)=-u(x, t) u_{x}(x, t)
$$

and

$$
f_{1}(x, t)=f(x, t)-e^{-x} f(0, t), \quad h_{1}(t)=h(t)-e^{-t} h(0) .
$$

This formula is obtained just as was (3.10) by viewing (1.1) as

$$
u_{t}+u_{x}+(v u)_{x}+\alpha u+u_{x x x}=g
$$

with the associated initial- and boundary-conditions, where $v=u / 2$ and $g \equiv 0$. Estimates of the various terms in (4.3) may be made exactly as in the proof of Proposition 3.11. The outcome of such an analysis is that given $T>0$, there are constants $C_{1}, C_{2}$ and $C_{3}$ such that for $0 \leq t \leq T$,

$$
\|u(\cdot, t)\|_{s} \leq C_{1} e^{-\alpha t}\|\phi\|_{s}+C_{2}\|u\|_{Y_{(0, T)}^{s}}^{2}+C_{3}|h|_{\frac{s+1}{3},(0, T)} .
$$

Here, $C_{1}$ and $C_{2}$ depend only on $s$ whilst $C_{3}$ depends on $s$ and $T$. Proposition 4.1 implies that there exists a $\delta>0$ and a constant $C_{4}$ depending only on $s$ and $T$ such that if

$$
\|(\phi, h)\|_{X_{(0, T)}^{s}} \leq \delta
$$

then

$$
\|u\|_{Y_{(0, T)}^{s}} \leq C_{4}\|(\phi, h)\|_{X_{(0, T)}^{s}} .
$$

In light of this, if (4.5) holds and (4.4) is evaluated at $t=T$, there obtains the inequality

$$
\|u(\cdot, T)\|_{s} \leq C_{1} e^{-\alpha T}\|\phi\|_{s}+C_{5}\|\phi\|_{s}^{2}+C_{5}|h|_{\frac{s+1}{3},(0, T)}^{2}+C_{3}|h|_{\frac{s+1}{3},(0, T)}
$$

where $C_{5}=2 C_{2} C_{4}$, say. Posit the restrictions

$$
\|\phi\|_{s} \leq \beta_{1} \quad \text { and } \quad|h|_{\frac{s+1}{3},(0, T)} \leq \beta_{2}
$$


where the positive parameters $\beta_{1}$ and $\beta_{2}$ will be specified momentarily. Choose $T>0$ so that

$$
C_{1} e^{-\alpha T} \leq \gamma<1
$$

Let $\omega=1 /(1-\gamma)$. With $T$ fixed, $C_{5}$ is fixed and we now choose $\beta_{1}$ and $\beta_{2}$ so that

$$
\beta_{1}+\beta_{2} \leq \delta
$$

and

$$
\omega\left[C_{5}\left(\beta_{1}^{2}+\beta_{2}^{2}\right)+C_{3} \beta_{2}\right] \leq \beta_{1} .
$$

(For example, first choose $\beta_{2}=\beta_{1} /\left(2 \omega C_{5}\right)$ and then take $\beta_{1} \leq 1 /\left(2 \omega C_{5}(1+\right.$ $\left.\left.1 /\left(4 \omega^{2} C_{5}^{2}\right)\right)\right)$ small enough that $\beta_{1}+\beta_{2} \leq \delta$.) For such values of $\beta_{1}$ and $\beta_{2}$, we have that

$$
\|u(\cdot, T)\|_{s}<\beta_{1}, \quad \text { and, by assumption, } \quad|h|_{\frac{s+1}{3},(T, 2 T)} \leq \beta_{2} .
$$

Hence, repeating the argument, it is determined that

$$
\sup _{T \leq t \leq 2 T}\|u(\cdot, t)\|_{s} \leq \beta_{1}
$$

Continuing inductively, it is adduced that

$$
\sup _{t \geq 0}\|u(\cdot, t)\|_{s} \leq \beta_{1}
$$

Let $y_{n}=u(\cdot, n T)$ for $n=0,1,2, \ldots$ Using the semigroup property of (1.1) and arguing as in the proof of (4.4) (see Proposition 3.11), one obtains constants $C_{1}, C_{2}$ and $C_{3}$ which are independent of $T$ and positive parameters $\beta_{1}$ and $\beta_{2}$ such that

$$
\left\|y_{n+1}\right\|_{s} \leq C_{1} e^{-\alpha T}\left\|y_{n}\right\|_{s}+C_{2}\left\|y_{n}\right\|_{s}^{2}+C_{3}|h|_{\frac{s+1}{3},(n T,(n+1) T)}
$$

for any $n \geq 1$ provided $\left\|y_{0}\right\|_{s} \leq \beta_{1}$ and

$$
\sup _{n \geq 0}|h|_{\frac{s+1}{3},(n T,(n+1) T)} \leq \beta_{2} .
$$

By Lemma 3.3, there exist $0<\nu<1, \beta_{1}^{*}>0$ and $\beta_{2}^{*}>0$ such that if $\left\|y_{0}\right\|_{s}<\beta_{1}^{*}$ and $b_{n}=C_{3}|h|_{(s+1) / 3,(n T,(n+1) T)} \leq \beta_{2}^{*}$ for all $n \geq 0$, then

$$
\left\|y_{n+1}\right\|_{s} \leq \nu^{n+1}\left\|y_{0}\right\|_{s}+\frac{b^{*}}{1-\nu}
$$

for $n=1,2, \ldots$, where $b^{*}=\max _{n}\left\{b_{n}\right\}$. This leads by standard arguments to the conclusion of Theorem 4.2 in the case that $3 / 4<s \leq 7 / 2$.

If $7 / 2<s \leq 13 / 2$, note that since

$$
u_{x x x}=-u_{t}-u u_{x}-u_{x}-\alpha u,
$$


it suffices to get an $H^{s-3}$-estimate of $u_{t}$ to obtain an $H^{s}$-estimate of $u$. To bound $u_{t}$, let $v=u_{t}$. Then $v$ solves the IBVP

$$
\left.\begin{array}{l}
v_{t}+v_{x}+(u v)_{x}+v_{x x x}+\alpha v=0, \quad x>0, \quad t>0, \\
v(x, 0)=\phi^{*}(x), \quad v(0, t)=h^{\prime}(t)
\end{array}\right\}
$$

where $\phi^{*}(x)=-\phi(x) \phi^{\prime}(x)-\alpha \phi(x)-\phi^{\prime}(x)-\phi^{\prime \prime \prime}(x)$. Because $s>7 / 2$, we know that $u \in C_{b}\left(0, \infty ; H^{3}\left(R^{+}\right)\right.$and there is $T>0$ and $\delta>0, r>0$ such that if

$$
\|\phi\|_{s}+\sup _{n \geq 0}|h|_{\frac{s+1}{3},(n T,(n+1) T)} \leq \delta,
$$

then

$$
\|u(\cdot, t)\|_{3} \leq C_{1} e^{-r t}\|\phi\|_{3}+C_{2} \sup _{n \geq 0}|h|_{\frac{4}{3},(n T,(n+1) T)}
$$

for any $t \geq 0$. By Proposition 4.1, there exists $C^{*}$ independent of $n$ such that

$$
\|u\|_{Y_{(n T,(n+1) T)}^{3}} \leq C^{*}\|(u(\cdot, n T), h)\|_{X_{(n T,(n+1) T)}^{3}} .
$$

Combining these yields

$$
\sup _{n \geq 0}\|u\|_{Y_{(n T,(n+1) T)}^{3}} \leq C^{*} C_{1}\|\phi\|_{3}+\left(C^{*}+C_{2}\right) \sup _{n \geq 0}|h|_{\frac{4}{3},(n T,(n+1) T)} .
$$

Applying Proposition 3.11, it is concluded that

$$
\|v(\cdot, t)\|_{s-3} \leq C_{1} e^{-r t}\left\|\phi^{*}\right\|_{s-3}+C_{2} \sup _{n \geq 0}\left|h^{\prime}\right|_{\frac{s-2}{3},(n T,(n+1) T)}
$$

for $t \geq 0$, or, because of the preceding remarks and the fact that $v=u_{t}$,

$$
\|u(\cdot, t)\|_{s} \leq C_{1} e^{-r t}\|\phi\|_{s}+C_{2} \sup _{n \geq 0}|h|_{\frac{s+1}{3},(n T,(n+1) T)}
$$

for $t \geq 0$ and $s \in(7 / 2,13 / 2]$. Thus, the inequality stated in the Theorem holds for $s \in(3 / 4,13 / 2]$. An inductive argument based on the idea just enunciated to extend $s \in(3 / 4,7 / 2]$ to $s \in(3 / 4,13 / 2]$ finishes the Proof of the Theorem.

In the last theorem, the $H^{s}\left(R^{+}\right)$-norm of the initial value $\phi$ was required to be small. The next task is to remove this smallness assumption. First, write the solution $u$ of (1.1) as

$$
u(x, t)=v(x, t)+w(x, t)
$$

where $v$ solves the linear problem

$$
\left.\begin{array}{l}
v_{t}+v_{x}+v_{x x x}+\alpha v=0, \quad \text { for } x, t \geq 0 \\
v(x, 0)=\phi_{1}(x), \quad v(0, t)=h(t)
\end{array}\right\}
$$

and $w$ solves

$$
\left.\begin{array}{l}
w_{t}+w_{x}+(v w)_{x}+w w_{x}+w_{x x x}+\alpha w=-v v_{x}, \quad x>0, \quad t>0, \\
w(x, 0)=\phi_{2}(x), \quad w(0, t)=0 .
\end{array}\right\}
$$


The auxiliary functions in (4.9) and (4.10) are $\phi_{1}(x)=e^{-x} \phi(0)$ and $\phi_{2}(x)=$ $\phi(x)-e^{-x} \phi(0)$. In the following propositions, the functions $\phi_{1}$ and $\phi_{2}$ are treated as general functions in the space $H^{s}\left(R^{+}\right)$. In this vein, the next result follows directly from Proposition 3.11.

Proposition 4.4. Let $s \in(3 / 4,7 / 2]$ be given. There exist positive constants $T$ and $r$ with $0<r<1$ and constants $C_{1}$ and $C_{2}$ such that for any $\phi_{1} \in H^{s}\left(R^{+}\right)$and $h \in H_{\mathrm{loc}}^{(s+1) / 3}\left(R^{+}\right)$satisfying $h(0)=\phi_{1}(0)$, the solution $v$ of $(4.9)$ satisfies

$$
\|v(\cdot, t)\|_{s} \leq C_{1} e^{-r t}\left\|\phi_{1}\right\|_{s}+C_{2} \sup _{n \geq 0}|h|_{\frac{s+1}{3},(n T,(n+1) T)}
$$

for any $t \geq 0$.

As regards the IBVP (4.10), the following result obtains and will be useful.

Proposition 4.5. Let $\beta=\sup _{t \geq 0}\left\|v_{x}(\cdot, t)\right\|_{L_{\infty}\left(R^{+}\right)}$and assume that

$$
r=\alpha-\beta>0 \text {. }
$$

Then the solution $w$ of (4.10) satisfies

$$
\|w(\cdot, t)\| \leq e^{-r t}\left\|\phi_{2}\right\|+\frac{\beta}{r} \sup _{0 \leq \tau \leq t}\|v(\cdot, \tau)\|
$$

for all $t \geq 0$.

Proof. Multiply both sides of the equation in (4.10) by $2 w$ and integrate over $R^{+}$ with respect to $x$. Integration by parts then leads to

$$
\begin{aligned}
& \frac{d}{d t} \int_{0}^{\infty} w^{2}(x, t) d x+2 \alpha \int_{0}^{\infty} w^{2}(x, t) d x+w_{x}^{2}(0, t) \\
& \quad=-\int_{0}^{\infty} v(x, t) v_{x}(x, t) w(x, t) d x-\int_{0}^{\infty} v_{x}(x, t) w^{2}(x, t) d x
\end{aligned}
$$

Straightforward estimates imply

$$
\frac{d}{d t}\|w(\cdot, t)\|+\alpha\|w(\cdot, t)\| \leq\left\|v_{x}(\cdot, t)\right\|_{L_{\infty}\left(R^{+}\right)}\|v(\cdot, t)\|+\left\|v_{x}(\cdot, t)\right\|_{L_{\infty}\left(R^{+}\right)}\|w(\cdot, t)\|
$$

from which (4.11) follows via Gronwall's Lemma.

Next, we derive an $H^{1}$-estimate of solutions $w$ of (4.10).

Proposition 4.6. There exist constants $\beta>0$ and $r$ with $0<r<1$ and nondecreasing continuous function $B_{j}: R^{+} \rightarrow R^{+}(j=1,2)$ such that if

$$
\sup _{t \geq 0}\|v(\cdot, t)\|_{2}<\beta,
$$


then the solution $w$ of (4.10) satisfies

$$
\|w(\cdot, t)\|_{1} \leq B_{1}\left(\left\|\phi_{2}\right\|\right) e^{-r t}\left\|\phi_{2}\right\|_{1}+B_{2}\left(\sup _{0 \leq \tau \leq t}\|v(\cdot, \tau)\|_{1}\right) \sup _{0 \leq \tau \leq t}\|v(\cdot, \tau)\|_{2}
$$

for any $t \geq 0$.

Proof. Multiply both sides of the equation in (4.10) by $2 w_{x x}+w^{2}$ and integrate over $R^{+}$with respect to $x$. Integration by parts then leads to the equation

$$
\begin{aligned}
\frac{d}{d t} \int_{0}^{\infty} & w_{x}^{2}(x, t) d x+2 \alpha \int_{0}^{\infty} w_{x}^{2}(x, t) d x+w_{x}^{2}(0, t)+w_{x x}^{2}(0, t) \\
= & \frac{1}{3} \frac{d}{d t} \int_{0}^{\infty} w^{3}(x, t) d x+\alpha \int_{0}^{\infty} w^{3}(x, t) d x+\frac{2}{3} \int_{0}^{\infty} w^{3}(x, t) v_{x}(x, t) d x \\
& -3 \int_{0}^{\infty} w_{x}^{2}(x, t) v_{x}(x, t) d x-2 \int_{0}^{\infty} w_{x}(x, t) w(x, t) v_{x x}(x, t) d x \\
& +\int_{0}^{\infty} v(x, t) v_{x}(x, t) w^{2}(x, t) d x-2 v(0, t) v_{x}(0, t) w_{x}(0, t) \\
& -2 \int_{0}^{\infty} v_{x}^{2}(x, t) w_{x}(x, t) d x-2 \int_{0}^{\infty} v(x, t) v_{x x}(x, t) w_{x}(x, t) d x
\end{aligned}
$$

Note that

$$
\begin{aligned}
& \int_{0}^{\infty} w_{x}^{2}(x, t) v_{x}(x, t) d x \leq\|v(\cdot, t)\|_{2}\|w(\cdot, t)\|_{1}^{2}, \\
& \int_{0}^{\infty} w_{x}(x, t) w(x, t) v_{x x}(x, t) d x \leq\|w(\cdot, t)\|_{L_{\infty}\left(R^{+}\right)}\left\|w_{x}(\cdot, t)\right\|\left\|v_{x x}(\cdot, t)\right\| \\
& \leq\|v(\cdot, t)\|_{2}\|w(\cdot, t)\|_{1}^{2} \\
& \int_{0}^{\infty} w^{3}(x, t) v_{x}(x, t) d x \leq\left\|v_{x}(\cdot, t)\right\|_{L_{\infty}\left(R^{+}\right)}\|w(\cdot, t)\|_{1}\|w(\cdot, t)\|^{2} \\
& \leq \frac{1}{2}\|v(\cdot, t)\|_{2}^{2}\|w(\cdot, t)\|_{1}^{2}+\frac{1}{2}\|w(\cdot, t)\|^{4}, \\
& \int_{0}^{\infty} v(x, t) v_{x}(x, t) w^{2}(x, t) d x \leq\|w(\cdot, t)\|\|w(\cdot, t)\|_{1}\|v(\cdot, t)\|_{1}\left\|v_{x}(\cdot, t)\right\| \\
& \leq\|v(\cdot, t)\|_{1}^{2}\|w(\cdot, t)\|_{1}^{2}, \\
& v(0, t) v_{x}(0, t) w_{x}(0, t) \leq \frac{1}{2} w_{x}^{2}(0, t)+\|v(\cdot, t)\|_{2}^{2}, \\
& \int_{0}^{\infty} v_{x}^{2}(x, t) w_{x}(x, t) d x \leq\left\|v_{x}(\cdot, t)\right\|_{L_{\infty}\left(R^{+}\right)}\left\|v_{x}(\cdot, t)\right\|\left\|w_{x}(\cdot, t)\right\| \\
& \leq\left\|v_{x}(\cdot, t)\right\|^{1 / 2}\left\|v_{x}(\cdot, t)\right\|\left\|v_{x x}(\cdot, t)\right\|^{1 / 2}\left\|w_{x}(\cdot, t)\right\| \\
& \leq \frac{1}{2}\|v(\cdot, t)\|_{2}\|w(\cdot, t)\|_{1}^{2}+\frac{1}{2}\left\|v_{x}(\cdot, t)\right\|^{3},
\end{aligned}
$$




$$
\begin{aligned}
\int_{0}^{\infty} w^{3}(x, t) d x & \leq\|w(\cdot, t)\|_{L_{\infty}\left(R^{+}\right)}\|w(\cdot, t)\|^{2} \\
& \leq\left\|w_{x}(\cdot, t)\right\|^{1 / 2}\|w(\cdot, t)\|^{5 / 2} \\
& \leq \epsilon\left\|w_{x}(\cdot, t)\right\|^{2}+C_{\epsilon}\|w(\cdot, t)\|^{10 / 3}
\end{aligned}
$$

and

$$
\begin{aligned}
\int_{0}^{\infty} v(x, t) v_{x x}(x, t) w_{x}(x, t) d x & \leq\|v(\cdot, t)\|_{L_{\infty}\left(R^{+}\right)}\left\|v_{x x}(\cdot, t)\right\|\left\|w_{x}(\cdot, t)\right\| \\
& \leq\left\|v_{x}(\cdot, t)\right\|^{1 / 2}\|v(\cdot, t)\|^{1 / 2}\left\|v_{x x}(\cdot, t)\right\|\left\|w_{x}(\cdot, t)\right\| \\
& \leq \frac{1}{2}\|v(\cdot, t)\|_{1}^{2}\|w(\cdot, t)\|_{1}^{2}+\frac{1}{2}\left\|v_{x x}(\cdot, t)\right\|^{2}
\end{aligned}
$$

Moreover, as in the proof of Proposition 4.6, it is seen that

$$
\begin{aligned}
& \frac{d}{d t} \int_{0}^{\infty} w^{2}(x, t) d x+2 \alpha \int_{0}^{\infty} w^{2}(x, t) d x \\
& \quad \leq\left(\left\|v_{x}\right\|_{L_{\infty}\left(R^{+}\right)}+\left\|v_{x}\right\|_{L_{\infty}\left(R^{+}\right)}^{2}\right) \int_{0}^{\infty} w^{2}(x, t) d x+\|v(\cdot, t)\|^{2}
\end{aligned}
$$

for any $t \geq 0$. Thus, there exist three constants $M_{j}(j=1,2,3)$ depending only on the positive parameter $\epsilon$ such that for all $t \geq 0$,

$$
\begin{aligned}
& \frac{d}{d t} \int_{0}^{\infty} w_{x}^{2}(x, t) d x+\alpha(2-\epsilon) \int_{0}^{\infty} w_{x}^{2}(x, t) d x \\
& \quad \leq M_{1}\left(\|v(\cdot, t)\|_{2}+\|v(\cdot, t)\|_{2}^{2}\right)\|w(\cdot, t)\|_{1}^{2}+M_{2}\|v(\cdot, t)\|_{2}^{2}\left(1+\|v(\cdot, t)\|_{1}\right) \\
& \quad+M_{3}\left(\|w(\cdot, t)\|^{4}+\|w(\cdot, t)\|^{10 / 3}\right)+\frac{1}{3} \frac{d}{d t} \int_{0}^{\infty} w^{3}(x, t) d x .
\end{aligned}
$$

Choose $\epsilon$ and $\beta$ small enough that if

$$
\sup _{t \geq 0}\|v(\cdot, t)\|_{2} \leq \beta
$$

then

$$
r_{1}=\alpha(2-\epsilon)-\mu_{1} \sup _{t \geq 0}\left(\|v(\cdot, t)\|_{2}+\|v(\cdot, t)\|_{2}^{2}\right)>0 .
$$

The preceding differential inequality then implies that

$$
\begin{aligned}
& \frac{d}{d t}\left(\|w(\cdot, t)\|_{1}^{2}-\frac{1}{3} \int_{0}^{\infty} w^{3}(x, t) d x\right)+r_{1}\left(\|w(\cdot, t)\|_{1}^{2}-\frac{1}{3} \int_{0}^{\infty} w^{3}(x, t) d x\right) \\
& \leq \frac{r_{1}}{3} \int_{0}^{\infty}|w(x, t)|^{3} d x+g_{1}(t)+g_{2}(t)
\end{aligned}
$$

where

$$
g_{1}(t)=M_{2}\|v(\cdot, t)\|_{2}^{2}\left(1+\|v(\cdot, t)\|_{1}\right)
$$


and

$$
g_{2}(t)=M_{3}\left(\|w(\cdot, t)\|^{4}+\|w(\cdot, t)\|^{10 / 3}\right) .
$$

In consequence, it is adduced that for all $t \geq 0$,

$$
\begin{aligned}
\|w(\cdot, t)\|_{H^{1}\left(R^{+}\right)}^{2} \leq & \frac{1}{3} \int_{0}^{\infty}|w(x, t)|^{3} d x+e^{-r_{1} t}\left(\left\|\phi_{2}\right\|_{s}+\frac{1}{3} \int_{0}^{\infty}\left|\phi_{2}(x)\right|^{3} d x\right) \\
& +\int_{0}^{t} e^{-r_{1}(t-\tau)}\left(\frac{r_{1}}{3} \int_{0}^{\infty}|w(x, \tau)|^{3} d x+g_{1}(\tau)+g_{2}(\tau)\right) d \tau .
\end{aligned}
$$

For $\epsilon>0$, there is a constant $C_{\epsilon}$ depending only on $\epsilon$ such that

$$
\begin{gathered}
\int_{0}^{\infty} w^{3}(x, t) d x \leq \epsilon\left\|w_{x}(\cdot, t)\right\|^{2}+C_{\epsilon}\|w(\cdot, t)\|^{10 / 3} \\
\int_{0}^{t} e^{-r_{1}(t-\tau)} \int_{0}^{\infty}|w(x, \tau)|^{3} d x \leq \frac{\epsilon}{r_{1}} \sup _{0 \leq \tau \leq t}\left\|w_{x}(\cdot, \tau)\right\|^{2}+\frac{C_{\epsilon}}{r_{1}} \sup _{0 \leq \tau \leq t}\|w(\cdot, \tau)\|^{10 / 3}
\end{gathered}
$$

and

$$
\int_{0}^{t} e^{-r_{1}(t-\tau)}\left(g_{1}(\tau)+g_{2}(\tau)\right) d \tau \leq \frac{1}{r_{1}} \sup _{0 \leq \tau \leq t}\left(g_{1}(\tau)+g_{2}(\tau)\right) .
$$

Combining those estimates with Proposition 4.6 yields the estimate (4.12).

The preceding propositions imply the following estimate for the solution of (1.1).

Theorem 4.7. There exist positive constants $T, \beta^{*}$ and $r$ and nondecreasing continuous functions $D_{j}: R^{+} \rightarrow R^{+}(j=1,2)$ such that if

$$
\sup _{n \geq 0}\|h\|_{H^{1}(n T,(n+1) T)} \leq \beta^{*}
$$

then the solution $u$ of (1.1) satisfies

$$
\begin{aligned}
\|u(\cdot, t)\|_{1} \leq & D_{1}(\|\phi\|) e^{-r t}\|\phi\|_{1} \\
& +D_{2}\left(\sup _{n \geq 0}|h|_{\frac{2}{3},(n T,(n+1) T)}\right) \sup _{n \geq 0}|h|_{1,(n T,(n+1) T)}
\end{aligned}
$$

for all $t \geq 0$.

Proof. By Proposition 4.4,

$$
\varlimsup_{t \rightarrow \infty}\|v(\cdot, t)\|_{2} \leq C_{2} \sup _{n \geq 0}|h|_{1,(n T,(n+1) T)} .
$$

For any particular positive value of $\beta$ in Proposition 4.6 , choose $\beta^{*}=\beta C_{2}$. Then, there exists $t_{0}>0$ such that

$$
\sup _{t \geq t_{0}}\|v(\cdot, t)\|_{2} \leq C_{2} \beta^{*} \leq \beta
$$


Because of Proposition 4.6, it is adduced that for all $t \geq t_{0}$,

$$
\|w(\cdot, t)\|_{1} \leq B_{1}\left(\left\|\phi_{2}\right\|\right) e^{-r t}\left\|w\left(\cdot, t_{0}\right)\right\|_{1}+B_{2}\left(\sup _{t_{0} \leq \tau \leq t} v(\cdot, \tau) \|_{1}\right) \sup _{t_{0} \leq \tau \leq t}\|v(\cdot, \tau)\|_{2} .
$$

Consequently, we have for $t \geq t_{0}$ that

$$
\|u(\cdot, t)\|_{1} \leq B_{1}\left(\left\|u\left(\cdot, t_{0}\right)\right\|\right) e^{-r t}\|\phi\|_{1}+B_{2}\left(\sup _{n \geq 0}|h|_{\frac{2}{3},(n T,(n+1) T)}\right) \sup _{n \geq 0}|h|_{1,(n T,(n+1) T)} .
$$

Thus (4.13) holds for appropriate choices of $D_{1}$ and $D_{2}$.

To conclude this section, an $H^{s}$-estimate $(3 \leq s \leq 4)$ is presented for solutions of (1.1).

Theorem 4.8. Let $s=3$, or 4 be given. There exist $T>0, r>0$ and $\beta>0$ such that for any s-compatible $(\phi, h) \in H^{s}\left(R^{+}\right) \times H_{\mathrm{loc}}^{(s+1) / 3}\left(R^{+}\right)$with

$$
\sup _{n \geq 0}|h|_{\frac{s+1}{3},(n T,(n+1) T)} \leq \beta,
$$

the corresponding solution $u$ of (1.1) satisfies

$$
\|u(\cdot, t)\|_{s} \leq C_{1} e^{-r t}\|\phi\|_{s}+C_{2} \sup _{n \geq 0}|h|_{\frac{s+1}{3},(n T,(n+1) T)}
$$

for all $t \geq 0$, where $C_{1}$ and $C_{2}$ depend only on $\|\phi\|_{1}$ and $\sup _{n \geq 0}|h|_{1,(n T,(n+1) T)}$.

Proof. Since

$$
u_{x x x}=-u_{t}-u u_{x}-u_{x}-\alpha u,
$$

it suffices to obtain an $L_{2^{-}}$or $H^{1}$-estimate of $u_{t}$ in order to derive an $H^{3}$ - or $H^{4}$ estimate of $u$. Let $v=u_{t}$. The function $v$ solves the IBVP

$$
\left.\begin{array}{l}
v_{t}+v_{x}+(u v)_{x}+v_{x x x}+\alpha v=0, \quad x>0, \quad t>0, \\
v(x, 0)=\phi^{*}(x), \quad v(0, t)=h^{\prime}(t)
\end{array}\right\}
$$

where $\phi^{*}(x)=-\phi(x) \phi^{\prime}(x)-\alpha \phi(x)-\phi^{\prime}(x)-\phi^{\prime \prime \prime}(x)$. By Proposition 3.11, there exist $T>0, r>0$ and $\tilde{\beta}>0$ such that if

$$
\sup _{n \geq 0}\|u\|_{Y_{(n T,(n+1) T)}^{s-3}} \leq \tilde{\beta}
$$

then

$$
\|v(\cdot, t)\|_{s-3} \leq C_{1} e^{-r t}\left\|\phi^{*}\right\|_{s-3}+C_{2} \sup _{n \geq 0}\left|h^{\prime}\right|_{(s-2) / 3,(n T,(n+1) T)}
$$

for all $t \geq 0$, which gives estimates of $u_{t}$. However, by Proposition 4.1,

$$
\|u\|_{Y_{(n T,(n+1) T)}^{s-3}} \leq\|u\|_{Y_{(n T,(n+1) T)}^{1}} \leq C\left(\|u(\cdot, n T)\|_{1}+|h|_{1,(n T,(n+1) T)}\right)
$$

if $\|u(\cdot, n T)\|_{1}+|h|_{1,(n T,(n+1) T)} \leq \delta_{T, 1}$. On the other hand, by Theorem 4.8, there is a nondecreasing continuous positive function $D_{2}: R^{+} \rightarrow R^{+}$such that

$$
\varlimsup_{t \rightarrow \infty}\|u(\cdot, t)\|_{1} \leq D_{2}\left(\sup _{n \geq 0}|h|_{\frac{2}{3},(n T,(n+1) T)}\right) \sup _{n \geq 0}|h|_{1,(n T,(n+1) T)} .
$$


Therefore, there exists a $t_{0}>0$ such that

$$
\left\|u\left(\cdot, t_{0}\right)\right\|_{1} \leq 2 D_{2}\left(\sup _{n \geq 0}|h|_{\frac{2}{3},(n T,(n+1) T)}\right) \sup _{n \geq 0}|h|_{1,(n T,(n+1) T)}
$$

for all $t \geq t_{0}$. We then consider (4.14) with the initial time taken to be $t=t_{0}$ instead of $t=0$. In this case the condition (4.15) is satisfied so long as the value of $\beta$ is chosen small enough. The proof is complete.

\section{Forced Oscillations and Their Stability}

In this section, attention is turned first to the pure boundary-value problem

$$
\left.\begin{array}{l}
u_{t}+u u_{x}+u_{x}+\alpha u+u_{x x x}=0, \quad x \in R^{+}, t \geq 0, \\
u(0, t)=h(t) .
\end{array}\right\}
$$

It is assumed that the boundary input $h(t)$ is periodic with period $\theta$ so that $h(t+\theta)=h(t)$ for all $t \geq 0$. We are concerned with whether or not this periodic forcing generates a time-periodic solution of (5.1). Note that, because of the dissipative term, it is not expected that the initial data will play any role in this question of long-time asymptotics.

Theorem 5.1. Let $s>3 / 4$ be given. There exists a $\beta>0$ such that if $h \in$ $H_{\mathrm{loc}}^{(s+1) / 3}\left(R^{+}\right)$is a periodic function of period $\theta$ satisfying

$$
|h|_{\frac{s+1}{3},(0, \theta)}<\beta,
$$

then (5.1) admits a solution $u^{*} \in C_{b}\left(0, \infty ; H^{s}\left(R^{+}\right)\right)$which is a time-periodic function of period $\theta$. Moreover, there exists a $\beta_{1}>0$ such that if $u_{1}^{*} \in C_{b}\left(0, \infty ; H^{s}\left(R^{+}\right)\right)$ is also a time-periodic solution of (5.1) with

$$
\left\|u_{1}^{*}(\cdot, 0)\right\|_{s} \leq \beta_{1}
$$

then

$$
u_{1}^{*}(x, t) \equiv u^{*}(x, t)
$$

for all $x, t \in R^{+}$.

Proof. Choose $\phi \in H^{s}\left(R^{+}\right)$which is $s$-compatible with $h$ and consider the IBVP

$$
\left.\begin{array}{l}
u_{t}+u_{x}+u u_{x}+u_{x x x}+\alpha u=0, \quad \text { for } x, t \geq 0, \\
u(x, 0)=\phi(x), \quad u(0, t)=h(t) .
\end{array}\right\}
$$

For the solution $u$ of $(5.2)$, let

$$
w(x, t)=u(x, t+\theta)-u(x, t) .
$$


Then, the new function $w$ solves the IBVP

$$
\left.\begin{array}{l}
w_{t}+w_{x}+(v w)_{x}+w_{x x x}+\alpha w=0, \quad x>0, \quad t>0, \\
w(x, 0)=\phi^{*}(x), \quad w(0, t)=0
\end{array}\right\}
$$

where $\phi^{*}(x)=u(x, \theta)-\phi(x)$ and $v(x, t)=\frac{1}{2}(u(x, t+\theta)+u(x, t))$. For this linearized system, the solution $w$ decays exponentially in time. More precisely, the following point is put forward.

Claim. There exist $T>0, r>0$ and $\beta>0$ such that if

$$
\|\phi\|_{s}+\sup _{n \geq 0}|h|_{\frac{s+1}{3},(n T,(n+1) T)} \leq \beta,
$$

then the solution $w$ of (5.3) satisfies

$$
\|w(\cdot, t)\|_{s} \leq C e^{-r t}\left\|\phi^{*}\right\|_{s}
$$

for all $t \geq 0$, where $C$ is independent of $\phi^{*}$ and $t$.

We only verify the claim for $3 / 4<s \leq 7 / 2$. When $s>7 / 2$, one may proceed by induction using the estimates of higher-order time derivatives, $w_{t}, w_{t t}, \ldots$, as in the proof of Theorem 4.8 .

By Proposition 3.11, there exist positive constants $T, \beta_{1}$ and $r$ such that

$$
\|w(\cdot, t)\|_{s} \leq C_{1} e^{-r t}
$$

for any $t \geq 0$ in case

$$
\sup _{n \geq 0}\|v\|_{Y_{(n T,(n+1) T)}^{s}} \leq \beta_{1} .
$$

To see (5.4) is satisfied, invoke Proposition 4.1 and Theorem 4.2. There exists $\delta>0$ such that if

$$
\|\phi\|_{s}+\sup _{n \geq 0}|h|_{\frac{s+1}{3},(n T,(n+1) T)}<\delta,
$$

then

$$
\begin{aligned}
\|v\|_{Y_{(n T,(n+1) T)}^{s}} \leq C_{2}\left(\|u(\cdot, n T)\|_{s}+|h|_{\frac{s+1}{3},(n T,(n+1) T)}\right) \\
\leq C\left(\|\phi\|_{s}+\sup _{n \geq 0}|h|_{\frac{s+1}{3},(n T,(n+1) T)}\right)
\end{aligned}
$$

for all $n \geq 0$. Consequently, inequality (5.4) is satisfied when $\delta$ is chosen small enough.

With this fact in hand, we show that (5.3) possesses a time-periodic solution of the same period $\theta$ as the boundary forcing. Denote by

$$
u_{n} \equiv u(x, n \theta)
$$


for $n \geq 1$. Write the difference $u_{n+m}-u_{n}$ in telescoping fashion to deduce

$$
\begin{aligned}
\left\|u_{n+m}-u_{n}\right\|_{s} & =\left\|\sum_{k=1}^{m} u_{n+k}-u_{n+k-1}\right\|_{s} \\
& \leq \sum_{k=1}^{m}\left\|u_{n+k}-u_{n+k-1}\right\|_{s} \\
& \leq \sum_{k=1}^{m}\|w(\cdot,(n+k-1) \theta)\|_{s} \\
& \leq \sum_{k=1}^{m} C e^{-(n+k-1) \theta}\left\|\phi^{*}\right\|_{s} \quad \text { (by the Claim) } \\
& \leq \frac{e^{-n \theta}}{1-e^{-\theta}} C\left\|\phi^{*}\right\|_{s}
\end{aligned}
$$

for any $m \geq 1$. Thus $\left\|u_{n+m}-u_{n}\right\|_{s}$ tends to zero uniformly in $m$ as $n \rightarrow \infty$, which is equivalent to saying that the sequence $\left\{u_{n}\right\}$ is a Cauchy sequence in $H^{s}\left(R^{+}\right)$. Let $\psi \in H^{s}\left(R^{+}\right)$be the limit of the sequence $\left\{u_{n}\right\}$, viz.

$$
\lim _{n \rightarrow \infty} u_{n}=\psi \quad \text { in } H^{s}\left(R^{+}\right) \text {. }
$$

By Theorem 4.8,

$$
\|\psi\|_{s} \leq C \sup _{n \geq 0}|h|_{\frac{s+1}{3},(n T,(n+1) T)} .
$$

Taking $\psi$ as an initial data together with the boundary input $h$ for $(5.2)$, it is asserted that the corresponding solution $u^{*}$ is a time-periodic solution of period $\theta$. To see this, note that while $u_{n}(\cdot)=u(\cdot, n \theta)$ converges to $\psi$ strongly in $H^{s}\left(R^{+}\right)$, it is also the case that $u(\cdot, n \theta+\theta)$ converges to $u^{*}(\cdot, \theta)$ strongly in the space $H^{s}\left(R^{+}\right)$ as $n \rightarrow \infty$ because of the continuity of the associated solution map. Observing that

$$
\begin{aligned}
& \left\|u^{*}(\cdot, \theta)-u^{*}(\cdot, 0)\right\|_{s} \leq\left\|u^{*}(\cdot, \theta)-u(\cdot, n \theta+\theta)\right\|_{s} \\
& \quad+\|u(\cdot, n \theta+\theta)-u(\cdot, n \theta)\|_{s}+\left\|u(\cdot, n \theta)-u^{*}(\cdot, 0)\right\|_{s}
\end{aligned}
$$

for any $n \geq 1$, it is concluded that

$$
u^{*}(\cdot, \theta)=u^{*}(\cdot, 0)
$$

and therefore that $u^{*}(x, t)$ is a time-periodic function of period $\theta$.

To demonstrate uniqueness, let $u_{1}$ be another time-periodic solution with the same boundary forcing $h$. Let $z(x, t)=u_{1}(x, t)-u^{*}(x, t)$. Then $z$ solves the linear problem (5.3) with $v=u_{1}+u^{*}$ and $\phi^{*}(x, t)=u_{1}(x, 0)-\psi(x)$. By Proposition 3.11, there exists a $\delta_{1}>0$ and $T>0$ such that if

$$
\sup _{n \geq 0}\|v\|_{Y_{(n T,(n+1) T)}^{s}} \leq \delta_{1}
$$


then $z$ decays to zero exponentially in the space $H^{s}\left(R^{+}\right)$, which in turn implies

$$
u_{1}(x, t)=u^{*}(x, t)
$$

for all $x \in R^{+}$and $t \geq 0$ because both of them are time-periodic functions. Remark that

$$
\begin{aligned}
\sup _{n \geq 0}\|v\|_{Y_{(n T,(n+1) T)}^{s}} & \leq \sup _{n \geq 0}\left\|u_{1}\right\|_{Y_{(n T,(n+1) T)}^{s}}+\sup _{n \geq 0}\left\|u^{*}\right\|_{Y_{(n T,(n+1) T)}^{s}} \\
& \leq C \sup _{n \geq 0}\left(\left\|u_{1}(\cdot, n T)\right\|_{s}+\left\|u^{*}(\cdot, n T)\right\|_{s}\right)+C \sup _{n \geq 0}|h|_{\frac{s+1}{3},(n T,(n+1) T)} \\
& \leq C\left(\left\|u_{1}(\cdot, 0)\right\|_{s}+\|\psi\|_{s}+|h|_{\frac{s+1}{3},(0, \theta)}\right) \\
& \leq C(\delta+\beta) \leq \delta_{1}
\end{aligned}
$$

if $\delta$ and $\beta$ are small enough. The proof is complete.

For a given periodic boundary forcing $h$ of period $\theta$, the IBVP (1.1) may be considered as a dynamical system in the infinite-dimensional space $H^{s}\left(R^{+}\right)$. It has just been shown that if the amplitude of $h$ is small, (1.1a) admits a unique time-periodic solution $u^{*}(x, t)$ of period $\theta$ satisfying the boundary condition $u^{*}(0, t)=h(t)$. Maintaining the dynamical systems perspective, one may view $u^{*}$ as a limit cycle of the dynamical system. A natural further inquiry is then to study the stability of this limit cycle.

Theorem 5.2. Under the assumptions of Theorem 5.1, the time-periodic solution $u^{*}$ is locally exponentially stable in the space $H^{s}\left(R^{+}\right)$, which is to say, there exist $\delta>0, r>0$ and $C>0$ such that for any given $\phi \in H^{s}\left(R^{+}\right)$, which satisfies

$$
\left\|\phi(\cdot)-u^{*}(\cdot, 0)\right\|_{s} \leq \delta
$$

and is s-compatible with $h$, the corresponding solution $u$ of (1.1) satisfies

$$
\left\|u(\cdot, t)-u^{*}(\cdot, t)\right\|_{s} \leq C e^{-r t}
$$

for any $t \geq 0$.

Proof. Let $w(x, t)=u(x, t)-u^{*}(x, t)$. Then $w$ solves

$$
\left.\begin{array}{l}
w_{t}+w_{x}+(v w)_{x}+w_{x x x}+\alpha w=0, \quad x>0, \quad t>0, \\
w(x, 0)=\phi_{1}, \quad w(0, t)=0
\end{array}\right\}
$$

where $v(x, t)=\frac{1}{2}\left(u(x, t)+u^{*}(x, t)\right)$ and $\phi_{1}(x)=\phi(x)-u^{*}(x, 0)$. As in the Proof of Theorem 5.1,

$$
\begin{aligned}
\sup _{n \geq 0}\|v\|_{Y_{(n T,(n+1) T)}^{s}} & \leq \sup _{n \geq 0}\|u\|_{Y_{(n T,(n+1) T)}^{s}}+\sup _{n \geq 0}\left\|u^{*}\right\|_{Y_{(n T,(n+1) T)}^{s}} \\
& \leq C \sup _{n \geq 0}\left(\|u(\cdot, n T)\|_{s}+\left\|u^{*}(\cdot, n T)\right\|_{s}\right)+C \sup _{n \geq 0}|h|_{\frac{s+1}{3},(n T,(n+1) T)}
\end{aligned}
$$




$$
\begin{aligned}
& \leq C\left(\|\phi\|_{s}+\|\psi\|_{s}+|h|_{\frac{s+1}{3},(0, \theta)}\right) \\
& \leq C\left(\|\phi-\psi\|_{s}+2\|\psi\|_{s}+|h|_{\frac{s+1}{3},(0, \theta)}\right) \\
& \leq C(\delta+\beta) .
\end{aligned}
$$

If $\delta$ and $\beta$ are small enough, it follows from Proposition 3.11 that

$$
\|w(\cdot, t)\|_{s} \leq C e^{-r t}\left\|\phi_{1}\right\|_{s}
$$

for any $t \geq 0$. The proof is complete.

The last two results are local: (i) uniqueness of time-periodic solutions holds under the assumption that the initial value is small; (ii) the time-periodic solution, considered as a limit cycle, is locally exponential stable. In a smoother space, the next result shows that uniqueness holds without the smallness assumption and that the unique limit cycle is, in fact, globally exponentially stable.

Theorem 5.3. Let $s$ be given such that $s=1$ or $3 k \leq s \leq 3 k+1$ for $k=1,2, \ldots$. Then there exists a $\beta>0$ such that if $h \in H_{\mathrm{loc}}^{\xi(s)}\left(R^{+}\right)$is a periodic function of period $\theta$ satisfying

$$
|h|_{\xi(s),(0, \theta)} \leq \beta
$$

where

$$
\xi(s)= \begin{cases}1 & \text { if } s=1, \\ (s+1) / 3 & \text { if } 3 k \leq s \leq 3 k+1 \text { for some positive interger } k,\end{cases}
$$

then (1.1a) admits a unique time-periodic solution $u^{*}$ of period $\theta$. Moreover, there exist $r>0$ and $C>0$ such that for any given $\phi \in H^{s}\left(R^{+}\right)$which is s-compatible with $h$, the corresponding solution $u$ of (1.1) satisfies

$$
\left\|u(\cdot, t)-u^{*}(\cdot, t)\right\|_{s} \leq C e^{-r t}
$$

for any $t \geq 0$.

Proof. By Theorem 4.8, there exist $T$ and $\beta_{2}$ such that if

$$
\sup _{n \geq 0}|h|_{\xi(s),(n T,(n+1) T)} \leq \beta_{2},
$$

then

$$
\varlimsup_{t \rightarrow \infty}\|u(\cdot, t)\|_{s} \leq C \sup _{n \geq 0}|h|_{\xi(s),(n T,(n+1) T)} .
$$

One may choose $\beta_{2}$ small enough such that $C \beta_{2}$ is smaller than $\beta$ and $\beta_{1}$ determined in Theorem 5.1. Thus there is a $T_{0}>0$ such that for any $t_{0} \geq T_{0},\left\|u\left(\cdot, t_{0}\right)\right\|_{s} \leq \beta_{2}$. Moreover, let $u^{*}$ be the time-periodic solution given in Theorem 5.1. We may choose $\beta_{2}$ and $\beta$ in Theorem 5.1 even smaller so that

$$
\left\|u\left(\cdot, t_{0}\right)-u^{*}(\cdot, 0)\right\|_{s} \leq \delta
$$


where $\delta$ is given in Theorem 5.2. Then by Theorem 5.2

$$
\left\|u\left(\cdot, t+t_{0}\right)-u^{*}(\cdot, t)\right\|_{s} \leq C e^{-r t}
$$

for all $t \geq 0$, which yields

$$
\left\|u(\cdot, t)-u^{*}(\cdot, t)\right\|_{s} \leq \tilde{C} e^{-r t}
$$

for all $t \geq 0$ since $u^{*}$ is a time-periodic function. This estimate implies both that the time-periodic solution of (5.1) is unique and that it is globally exponentially stable. The proof is complete.

\section{Conclusion}

While parts of the preceding developments are somewhat technical, the upshot is quite simple to describe. Consider a semi-infinite medium $\{x: x \geq 0\}$ in which wave propagation is governed by the damped Korteweg-de Vries equation

$$
u_{t}+u_{x}+u u_{x}+u_{x x x}+\alpha u=0 .
$$

Imagine this medium forced periodically from its finite end $x=0$, thus imposing upon (6.1) the boundary condition

$$
u(0, t)=h(t)
$$

for $t \geq 0$, where $h$ is periodic, or asymptotically periodic. The conclusion in view is that at least if the forcing is not too large, an assumption very much in line with derivations of the Korteweg-de Vries equation as a model of physical phenomena, the solution $u$ of $(6.1)-(6.2)$ with any initial state $u(x, 0)$ of the medium, settles down uniformly in $x$ to being periodic of the same period as that of boundary forcing $h$. Moreover, the solution $u$ of the initial-boundary-value problem converges exponentially rapidly to a solution of $(6.1)-(6.2)$ that is exactly time periodic. This time-periodic solution is unique and exponentially stable.

It would be interesting to have similar theory for a wider range of dissipative mechanisms and for other equations besides the Korteweg-de Vries equation. Similar results appear to be valid without dissipation, but of course, in the absence of suitably strong dissipation, the convergence to periodicity is only uniform on compact sets.

\section{Acknowledgment}

The authors wish to acknowledge the manuscript of Bona and $\mathrm{Wu}[3]$ concerned with related linear and nonlinear problems that inspired some of the calculations in Sec. 3. 


\section{References}

[1] J. L. Bona, W. G. Pritchard and L. R. Scott, An evaluation of a model equation for water waves, Philos. Trans. Royal Soc. London Series A 302 (1981) 457-510.

[2] J. L. Bona, S. M. Sun and B.-Y. Zhang, A non-homogeneous boundary-value problem for the Korteweg-de Vries equation in a quarter plane, Trans. Amer. Math. Soc. 354 (2002) 427-490.

[3] J. L. Bona and J. Wu, Temporal growth and eventual periodicity for dispersive wave equations in a quarter plane, preprint, 2002.

[4] J. Bourgain, Construction of periodic solutions of nonlinear wave equations in higher dimension, Geom. Funct. Anal. 5 (1995) 105-140.

[5] H. Brézis, Periodic solutions of nonlinear vibrating strings and duality principles, Bull. Amer. Math. Soc. 8 (1983) 409-425.

[6] W. Craig and C. E. Wayne, Newton's method and periodic solutions of nonlinear wave equations, Comm. Pure Appl. Math. 46 (1993) 1409-1498.

[7] J. B. Keller and L. Ting, Periodic vibrations of systems governed by nonlinear partial differential equations, Comm. Pure and Appl. Math. 19 (1966) 371-420.

[8] J. U. Kim, Forced vibration of an aero-elastic plate, J. Math. Anal. Appl. 113 (1986) 454-467.

[9] J.-M. Ghidaglia, Weakly damped forced Korteweg-de Vries equations behave as a finite-dimensional dynamical system in the long time, J. Differential Equations $\mathbf{7 4}$ (1988) 369-390.

[10] J.-M. Ghidaglia, A note on the strong convergence towards attractors of damped forced KdV equations, J. Differential Equations 110 (1994) 356-359.

[11] J.-L. Lion and E. Magenes, Non-Homogeneous Boundary Value Problems and Applications, Vol. 1, Springer-Verlag, Heidelberg, 1972.

[12] P. H. Rabinowitz, Periodic solutions of nonlinear hyperbolic differential equations, Comm. Pure Appl. Math. 20 (1967) 145-205.

[13] P. H. Rabinowitz, Free vibrations for a semi-linear wave equation, Comm. Pure Appl. Math. 31 (1978) 31-68.

[14] G. R. Sell and Y. C. You, Inertial manifolds: the nonselfadjoint case, J. Differential Equations 96 (1992) 203-255.

[15] O. Vejvoda, Partial Differential Equations: Time-Periodic Solutions, Martinus Nijhoff Publishers, 1982.

[16] C. E. Wayne, Periodic solutions of nonlinear partial differential equations, Notices Amer. Math. Soc. 44 (1997) 895-902.

[17] C. E. Wayne, Periodic and quasi-periodic solutions of nonlinear wave equations via KAM theory, Comm. Math. Phys. 127 (1990) 479-528.

[18] B.-Y. Zhang, Forced oscillations of a regularized long-wave equation and their global stability: Differential Equations and Computational Simulations (Chengdu, 1999), World Scientific Publishing, River Edge, NJ, 2000, pp. 456-463.

[19] B.-Y. Zhang, Forced oscillation of the Korteweg-de Vries-Burgers equation and its stability, Control of Nonlinear Distributed Parameter Systems, College Station, TX, 1999, Lecture Notes in Pure and Appl. Math., 218, Dekker, New York, 2001, pp. $337-357$. 